\title{
NEAR- AND MID-INFRARED PHOTOMETRY OF THE PLEIADES AND A NEW LIST OF SUBSTELLAR CANDIDATE MEMBERS ${ }^{1,2}$
}

\author{
John R. STAufFer \\ Spitzer Science Center, Caltech 314-6, Pasadena, CA 91125; stauffer@ipac.caltech.edu \\ LeE W. Hartmann \\ Astronomy Department, University of Michigan \\ Giovanni G. Fazio, Lori E. Allen, and Brian M. Patten \\ Harvard-Smithsonian Center for Astrophysics, 60 Garden Street, Cambridge, MA 02138 \\ Patrick J. Lowrance, Robert L. Hurt, and Luisa M. Rebull \\ Spitzer Science Center, Caltech, Pasadena, CA 91125 \\ Roc M. Cutri and Solange V. Ramirez \\ Infrared Processing and Analysis Center, Caltech 220-6, Pasadena, CA 91125 \\ Erick T. Young, George H. Rieke, Nadya I. Gorlova, ${ }^{3}$ and James C. Muzerolle \\ Steward Observatory, University of Arizona, Tucson, AZ 85726 \\ Cathy L. Slesnick \\ Astronomy Department, Caltech, Pasadena, CA 91125 \\ AND \\ Michael F. SKRUTSKIE \\ Astronomy Department, University of Virginia, Charlottesville, VA 22903 \\ Received 2007 February 23; accepted 2007 April 6
}

\begin{abstract}
We make use of new near- and mid-IR photometry of the Pleiades cluster in order to help identify proposed cluster members. We also use the new photometry with previously published photometry to define the single-star mainsequence locus at the age of the Pleiades in a variety of color-magnitude planes. The new near- and mid-IR photometry extend effectively 2 mag deeper than the 2MASS All-Sky Point Source catalog, and hence allow us to select a new set of candidate very low-mass and substellar mass members of the Pleiades in the central square degree of the cluster. We identify 42 new candidate members fainter than $K_{s}=14$ (corresponding to $0.1 M_{\odot}$ ). These candidate members should eventually allow a better estimate of the cluster mass function to be made down to of order $0.04 M_{\odot}$. We also use new IRAC data, in particular the images obtained at $8 \mu \mathrm{m}$, in order to comment briefly on interstellar dust in and near the Pleiades. We confirm, as expected, that - with one exception - a sample of low-mass stars recently identified as having $24 \mu \mathrm{m}$ excesses due to debris disks do not have significant excesses at IRAC wavelengths. However, evidence is also presented that several of the Pleiades high-mass stars are found to be impacting with local condensations of the molecular cloud that is passing through the Pleiades at the current epoch.
\end{abstract}

Subject headings: open clusters and associations: individual (Pleiades) - stars: low-mass, brown dwarfs

Online material: color figure, machine-readable tables

\section{INTRODUCTION}

Because of its proximity, youth, richness, and location in the northern hemisphere, the Pleiades has long been a favorite target of observers. The Pleiades was one of the first open clusters to have members identified via their common proper motion (Trumpler 1921), and the cluster has since then been the subject of more than a dozen proper-motion studies. Some of the earliest photoelectric

\footnotetext{
${ }^{1}$ This work is based (in part) on observations made with the Spitzer Space Telescope, which is operated by the Jet Propulsion Laboratory, California Institute of Technology, under NASA contract 1407.

2 This publication makes use of data products from the Two Micron All Sky Survey, which is a joint project of the University of Massachusetts and the Infrared Processing and Analysis Center/California Institute of Technology, funded by the National Aeronautics and Space Administration and the National Science Foundation.

${ }^{3}$ Current address: University of Florida, 211 Bryant Space Center, Gainesville, FL 32611.
}

photometry was for members of the Pleiades (Cummings 1921), and the cluster has been the subject of dozens of papers providing additional optical photometry of its members. The youth and nearness of the Pleiades make it a particularly attractive target for identifying its substellar population, and it was the first open cluster studied for those purposes (Jameson \& Skillen 1989; Stauffer et al. 1989). More than 20 papers have been subsequently published, identifying additional substellar candidate members of the Pleiades or studying their properties.

We have three primary goals for this paper. First, while extensive optical photometry for Pleiades members is available in the literature, photometry in the near- and mid-IR is relatively spotty. We will remedy this situation by using new 2MASS $J H K_{s}$ and Spitzer Infrared Array Camera (IRAC) photometry for a large number of Pleiades members. We will use these data to help identify cluster nonmembers and to define the single-star locus in colormagnitude diagrams for stars of $100 \mathrm{Myr}$ age. Second, we will use 


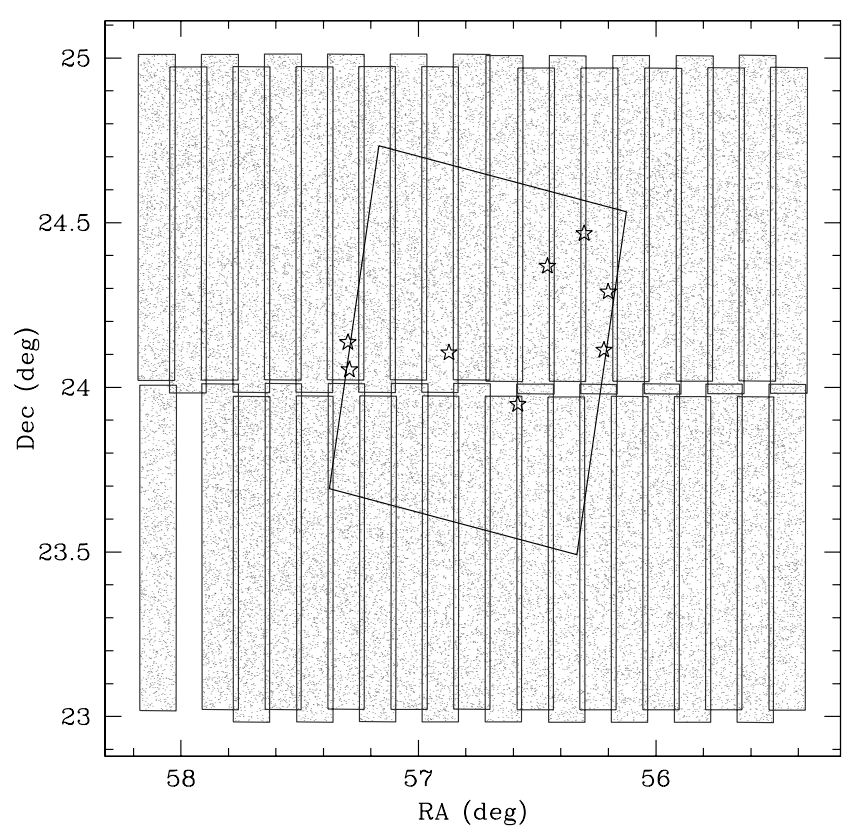

FIG. 1.- Spatial coverage of the 6 times deeper "2MASS $6 x$ " observations of the Pleiades. The 2MASS survey region is approximately centered on Alcyone, the most massive member of the Pleiades. The trapezoidal box roughly indicates the region covered with the shallow IRAC survey of the cluster core. The star symbols correspond to the brightest B star members of the cluster. The gray points are the location of objects in the 2MASS 6x Point Source Catalog. [See the electronic edition of the Supplement for a color version of this figure.]

our new IR imaging photometry of the center of the Pleiades to identify a new set of candidate substellar members of the cluster, extending down to stars expected to have masses of order $0.04 M_{\odot}$. Third, we will use the IRAC data to briefly comment on the presence of circumstellar debris disks in the Pleiades and the interaction of the Pleiades stars with the molecular cloud that is currently passing through the cluster.

In order to make best use of the IR imaging data, we will begin with a necessary digression. As noted above, more than a dozen proper-motion surveys of the Pleiades have been made in order to identify cluster members. However, no single catalog of the cluster has been published that attempts to collect all of those candidate members in a single table and cross-identify those stars. Another problem is that, while there have been many papers devoted to providing optical photometry of cluster members, that photometry has been bewilderingly inhomogeneous in terms of the number of photometric systems used. In $\S 3$ and in the Appendix, we describe our efforts to create a reasonably complete catalog of candidate Pleiades members and to provide optical photometry transformed to the best of our ability onto a single system.

\section{NEW OBSERVATIONAL DATA}

\section{1. $2 M A S S$ " $6 x$ ” Imaging of the Pleiades}

During the final months of Two Micron All Sky Survey (2MASS; Skrutskie et al. 2006) operations, a series of special observations were carried out that employed exposures 6 times longer than used for the primary survey. These so-called " $6 \mathrm{x}$ " observations targeted 30 regions of scientific interest including a $3^{\circ} \times 2^{\circ}$ area centered on the Pleiades cluster. The 2MASS $6 x$ data were reduced using an automated processing pipeline similar to that used for the main survey data, and a calibrated $6 \mathrm{x}$ Image Atlas and extracted 6x Point and Extended Source Catalogs

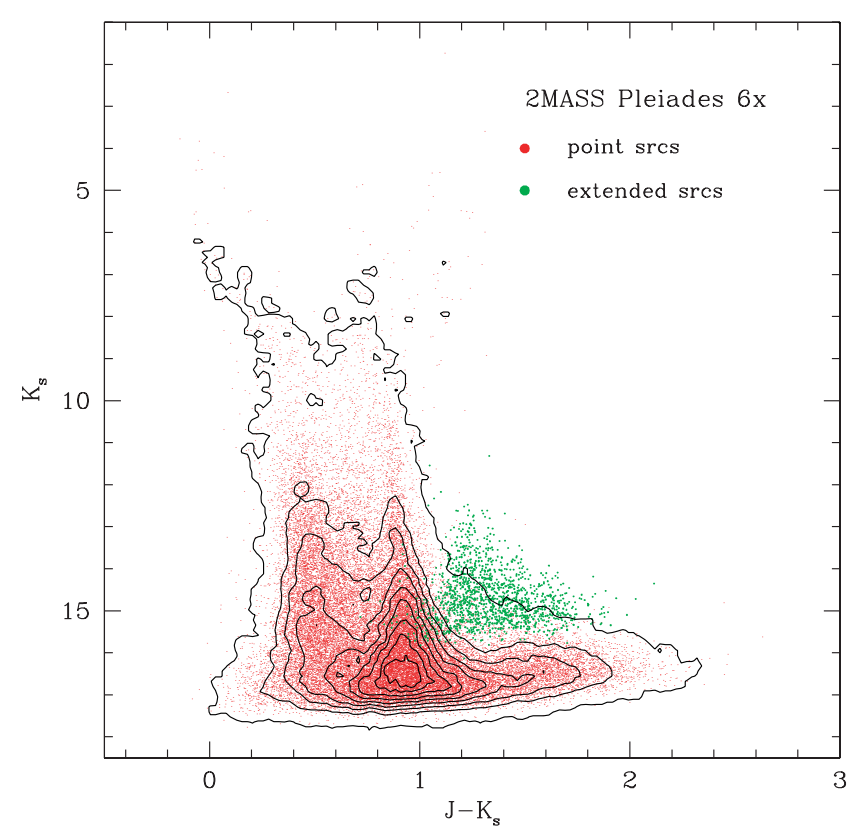

FIG. 2.-Color-magnitude diagram for the Pleiades derived from the 2 MASS $6 \mathrm{x}$ observations. The red dots correspond to objects identified as unresolved, whereas the green dots correspond to extended sources (primarily background galaxies). The lack of green dots fainter than $K=16$ is indicative that too few photons are available to identify sources as extended - the extragalactic population presumably increases to fainter magnitudes.

(6x-PSC and 6x-XSC) analogous to the 2MASS All-Sky Atlas, PSC, and XSC have been released as part of the 2MASS Extended Mission. A description of the content and formats of the $6 \mathrm{x}$ image and catalog products, and details about the $6 x$ observations and data reduction, are given in $\S$ A3 of the 2MASS Explanatory Supplement by Cutri et al. ${ }^{4}$ The 2 MASS $6 x$ Atlas and Catalogs may be accessed via the online services of the NASA/IPAC Infrared Science Archive. ${ }^{5}$

Figure 1 shows the area on the sky imaged by the 2MASS $6 x$ observations in the Pleiades field. The region was covered by two rows of scans, each scan being $1^{\circ}$ long (in declination) and $8.5^{\prime}$ wide in right ascension. Within each row, the scans overlap by approximately $1^{\prime}$ in right ascension. There are small gaps in coverage in the declination boundary between the rows, and one complete scan in the southern row is missing because the data in that scan did not meet the minimum required photometric quality. The total area covered by the 6x Pleiades observations is approximately $5.3 \mathrm{deg}^{2}$.

There are approximately 43,000 sources extracted from the $6 \mathrm{x}$ Pleiades observations in the 2MASS 6x-PSC, and nearly 1500 in the 6x-XSC. Because there are at most about 1000 Pleiades members expected in this region, only $\sim 2 \%$ of the $6 x$-PSC sources are cluster members, and the rest are field stars and background galaxies. The $6 \mathrm{x}-\mathrm{XSC}$ objects are virtually all resolved background galaxies. Near-infrared color-magnitude and color-color diagrams of the unresolved sources from the 2MASS 6x-PSC and all sources in the $6 \mathrm{x}-\mathrm{XSC}$ sources from the Pleiades region are shown in Figures 2 and 3, respectively. The extragalactic sources tend to be redder than most stars, and the galaxies become relatively more numerous toward fainter magnitudes. Unresolved galaxies dominate the point sources that are fainter than $K_{s}>15.5$ and redder than $J-K_{s}>1.2 \mathrm{mag}$.

\footnotetext{
${ }_{5}^{4}$ See http://www.ipac.caltech.edu/2mass/releases/allsky/doc/explsup.html.

${ }^{5}$ See http://irsa.ipac.caltech.edu.
} 


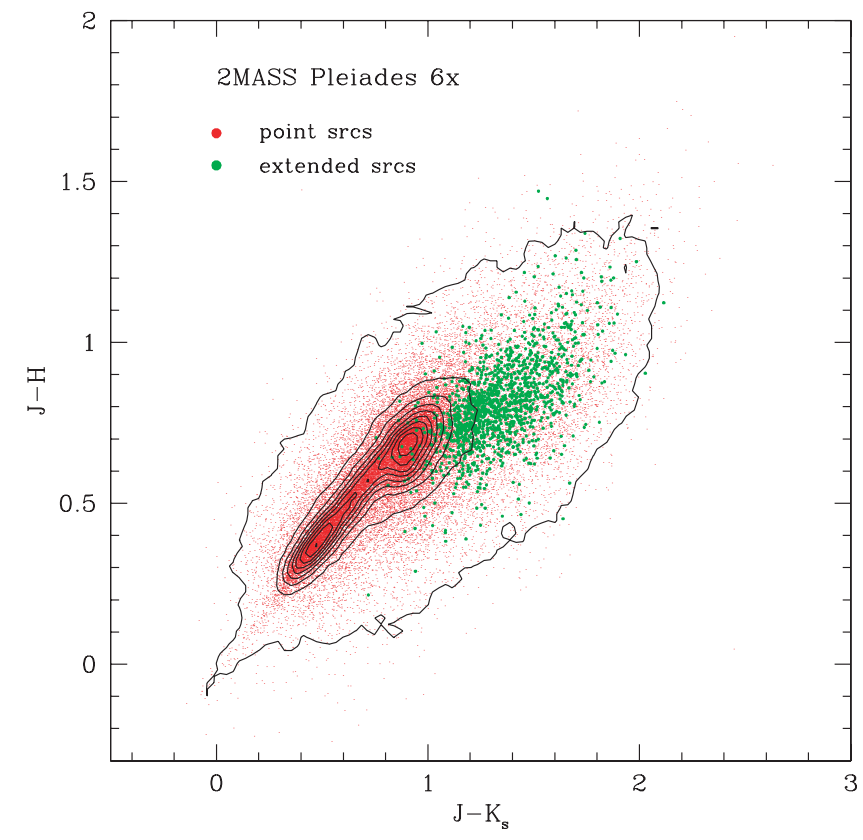

Fig. 3.- Same as Fig. 2, except in this case the axes are $J-H$ and $H-K_{s}$. The extragalactic objects are very red in both colors.

The 2MASS $6 \mathrm{x}$ observations were conducted using the same freeze-frame scanning technique used for the primary survey (Skrutskie et al. 2006). The longer exposure times were achieved by increasing the "READ2-READ1" integration to $7.8 \mathrm{~s}$ from the $1.3 \mathrm{~s}$ used for primary survey. However, the $51 \mathrm{~ms}$ "READ1" exposure time was not changed for the 6x observations. As a result, there is an effective "sensitivity gap" in the 8-11 mag region where objects may be saturated in the $7.8 \mathrm{~s}$ READ2-READ1 $6 \mathrm{x}$ exposures, but too faint to be detected in the $51 \mathrm{~ms}$ READ1 exposures. Because the sensitivity gap can result in incompleteness and/or flux bias in the photometric overlap regime, the nearinfrared photometry for sources brighter than $J=11$ mag in the 6x-PSC was taken from the 2MASS All-Sky PSC during compilation of the catalog of Pleiades candidate members presented in Table 2 (see $\S 3)$.

\subsection{Shallow IRAC Imaging}

Imaging of the Pleiades with Spitzer was obtained in 2004 April as part of a joint GTO program conducted by the IRAC instrument team and the Multiband Imaging Photometer for Spitzer (MIPS) instrument team. Initial results of the MIPS survey of the Pleiades have already been reported in Gorlova et al. (2006). The IRAC observations were obtained as two astronomical observing requests (AORs). One of them was centered near the cluster center, at R.A. $=03^{\mathrm{h}} 47^{\mathrm{m}} 00.0^{\mathrm{s}}$ and decl. $=24^{\circ} 07^{\prime}(\mathrm{J} 2000.0)$, and consisted of a 12 row by 12 column map, with "frame times" of 0.6 and $12.0 \mathrm{~s}$ and two dithers at each map position. The map steps were $290^{\prime \prime}$ in both the column and row direction. The resultant map covers a region of approximately $1 \mathrm{deg}^{2}$, and a total integration time per position of $24 \mathrm{~s}$ over most of the map. The second AOR used the same basic mapping parameters, except it was smaller ( 9 rows by 9 columns) and was instead centered northwest from the cluster center at R.A. $=03^{\mathrm{h}} 44^{\mathrm{m}} 36.0^{\mathrm{s}}$ and decl. $=$ $25^{\circ} 24^{\prime}$. A two-band color image of the AOR covering the center of the Pleiades is shown in Figure 4. A pictorial guide to the IRAC image providing Greek names for a few of the brightest stars, and Hertzsprung (1947) numbers for several stars mentioned in $\S 6$ is provided in Figure 5.

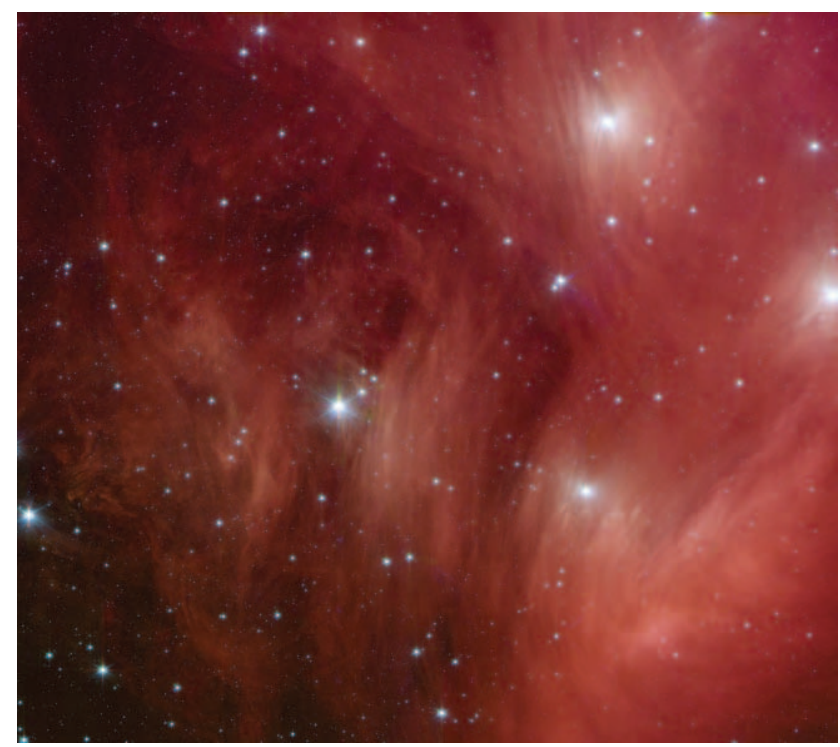

FIG. 4.-Two-color $(4.5$ and $8.0 \mu \mathrm{m})$ mosaic of the central square degree of the Pleiades from the IRAC survey. North is approximately vertical, and east is approximately to the left. The bright star nearest the center is Alcyone; the bright star at the left of the mosaic is Atlas; and the bright star at the right of the mosaic is Electra.

We began our analysis with the basic calibrated data (BCDs) from the Spitzer pipeline, using the S13 version of the Spitzer Science Center pipeline software. Artifact mitigation and masking was done using the IDL tools provided on the Spitzer contributed software Web site. For each AOR, the artifact-corrected BCDs were combined into single mosaics for each channel using the post-BCD "MOPEX" package (Makovoz \& Marleau 2005). The mosaic images were constructed with $1.22^{\prime \prime} \times 1.22^{\prime \prime}$ pixels (i.e., approximately the same pixel size as the native IRAC arrays).

We derived aperture photometry for stars present in these IRAC mosaics using both APEX (a component of the MOPEX package) and the "phot" routine in DAOPHOT. In both cases, we used a 3 pixel radius aperture and a sky annulus from 3 to 7 pixels (except that for channel 4 , for the phot package we used a 2 pixel

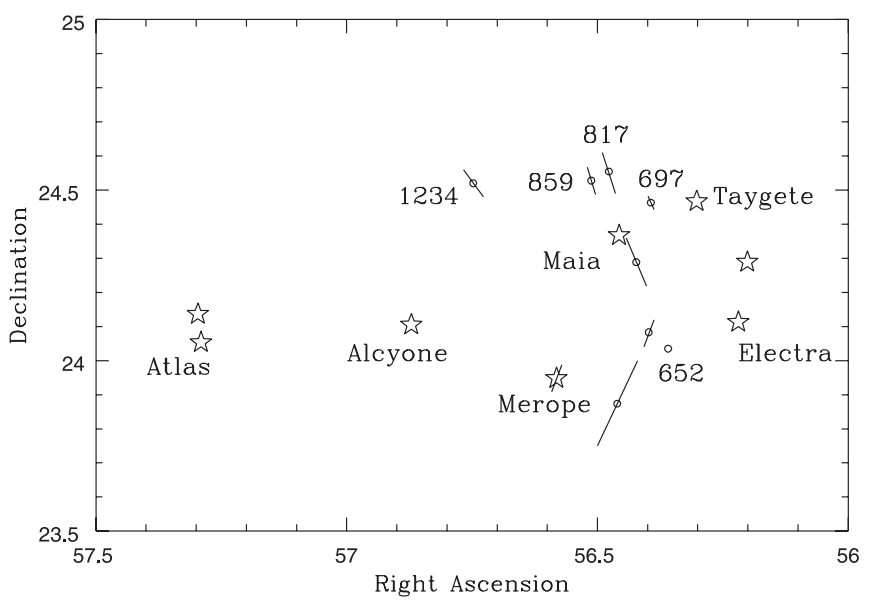

FIG. 5.-Finding chart corresponding approximately to the region imaged with IRAC. The large, five-pointed stars are all of the Pleiades members brighter than $V=5.5$. The small open circles correspond to other cluster members. Several stars with $8 \mu \mathrm{m}$ excesses are labeled by their HII numbers and are discussed further in $\S 6$. The short lines through several of the stars indicate the size and position angle of the residual optical polarization (after subtraction of a constant foreground component), as provided in Fig. 6 of Breger (1986). 


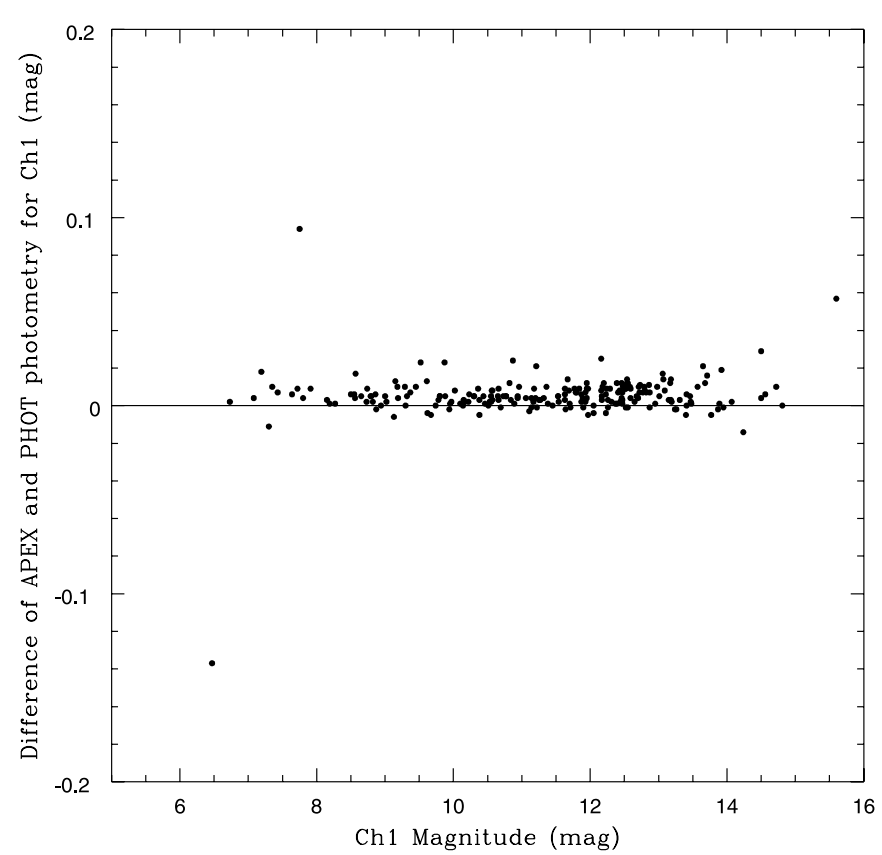

FIG. 6.-Comparison of aperture photometry for Pleiades members derived from the IRAC 3.6 $\mu \mathrm{m}$ mosaic using the Spitzer APEX package and the IRAF implementation of DAOPHOT.

radius aperture and a 2-6 pixel annulus because that provided more reliable fluxes at low flux levels). We used the flux for zeromagnitude calibrations provided in the IRAC data handbook (280.9, 179.7, 115.0, and 64.1 Jy for channels $1-4$, respectively), and the aperture corrections provided in the same handbook (multiplicative flux correction factors of 1.124, 1.127, 1.143, and 1.584 for channels $1-4$, inclusive. The channel 4 correction factor is much bigger because it is for an aperture radius of 2 rather than 3 pixels.).

Figures 6 and 7 provide two means to assess the accuracy of the IRAC photometry. The first figure compares the aperture photometry from APEX to that from phot and shows that the two packages yield very similar results when used in the same way. For this reason, we have simply averaged the fluxes from the two packages to obtain our final reported value. The second figure shows the difference between the derived 3.6 and $4.5 \mu \mathrm{m}$ magnitudes for Pleiades members. Based on previous studies (e.g., Allen et al. 2004), we expected this difference to be essentially zero for most stars, and the Pleiades data corroborate that expectation. For $[3.6]<10.5$, the rms dispersion of the magnitude difference between the two channels is $0.024 \mathrm{mag}$. Assuming that each channel has similar uncertainties, this indicates an internal $1 \sigma$ accuracy of order $0.017 \mathrm{mag}$. The absolute calibration uncertainty for the IRAC fluxes is currently estimated at of order $0.02 \mathrm{mag}$. Figure 7 also shows that fainter than $[3.6]=10.5$ (spectral type later than about M0), the [3.6] - [4.5] color for M dwarfs departs slightly from zero, becoming increasingly redder to the limit of the data (about M6).

\section{A CATALOG OF PLEIADES CANDIDATE MEMBERS}

If one limits oneself to only stars visible with the naked eye, it is easy to identify which stars are members of the Pleiades - all of the stars within a degree of the cluster center that have $V<6$ are indeed members. However, if one were to try to identify the M dwarf stellar members of the cluster (roughly $14<V<23$ ), only of order $1 \%$ of the stars toward the cluster center are likely

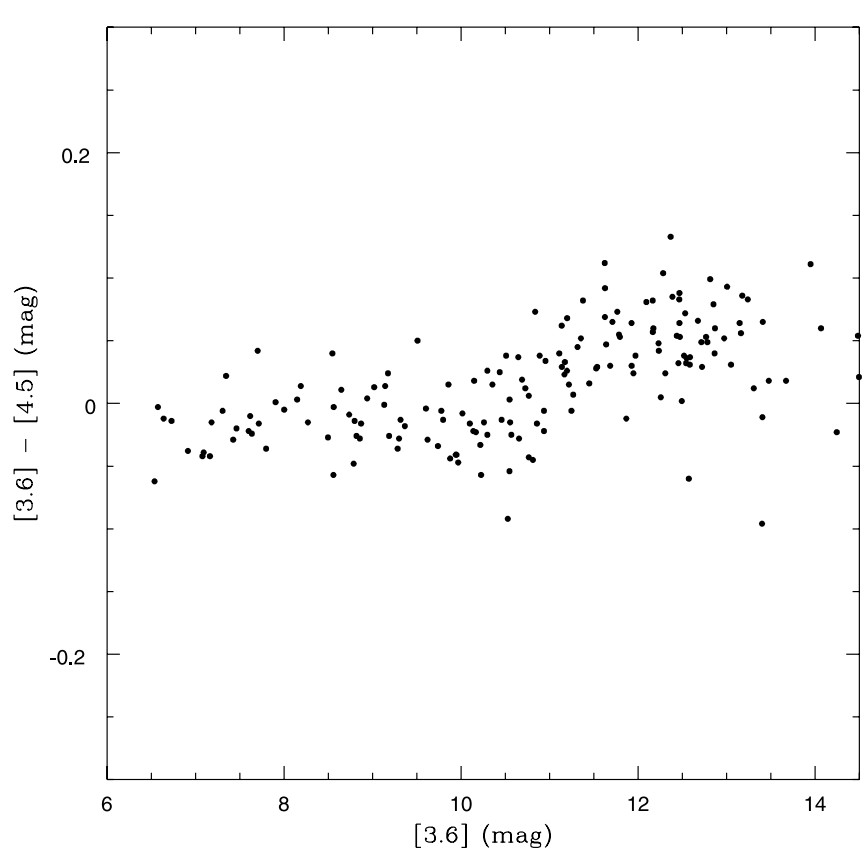

FIG. 7.-Difference between aperture photometry for Pleiades members for IRAC channels 1 and 2. The [3.6] - [4.5] color begins to depart from essentially zero at magnitudes of $\sim 10.5$, corresponding approximately to spectral type $\mathrm{M} 0$ in the Pleiades.

to be members, and it is much harder to construct an uncontaminated catalog. The problem is exacerbated by the fact that the Pleiades is old enough that mass segregation through dynamical processes has occurred, and therefore one has to survey a much larger region of the sky in order to include all of the M dwarf members.

The other primary difficulty in constructing a comprehensive member catalog for the Pleiades is that the pedigree of the candidates varies greatly. For the best-studied stars, astrometric positions can be measured over temporal baselines ranging up to a century or more, and the separation of cluster members from field stars in a vector point diagram (VPD) can be extremely good. In addition, accurate radial velocities and other spectral indicators are available for essentially all of the bright cluster members, and these further allow membership assessment to be essentially definitive. Conversely, at the faint end (for stars near the hydrogenburning mass limit in the Pleiades), members are near the detection limit of the existing wide-field photographic plates, and the errors on the proper motions become correspondingly large, causing the separation of cluster members from field stars in the VPD to become poor. These stars are also sufficiently faint that spectra capable of discriminating members from field dwarfs can only be obtained with $8 \mathrm{~m}$ class telescopes, and only a very small fraction of the faint candidates have had such spectra obtained. Therefore, any comprehensive catalog created for the Pleiades will necessarily have stars ranging from certain members to candidates for which very little is known and where the fraction of spurious candidate members increases to lower masses.

In order to address the membership uncertainties and biases, we have chosen a sliding scale for inclusion in our catalog. For all stars, we require that the available photometry yields location in color-color and color-magnitude diagrams consistent with cluster membership. For the stars with well-calibrated photoelectric photometry, this means the star should not fall below the Pleiades single-star locus by more than about 0.2 mag or above that locus by more than about $1.0 \mathrm{mag}$ (the expected displacement for a 
TABLE 1

Pleiades Membership Surveys Used as Sources

\begin{tabular}{|c|c|c|c|c|}
\hline Reference & $\begin{array}{l}\text { Area Covered } \\
\left(\operatorname{deg}^{2}\right)\end{array}$ & $\begin{array}{l}\text { Magnitude Range } \\
\text { (and Band) }\end{array}$ & $\begin{array}{l}\text { Number of } \\
\text { Candidates }\end{array}$ & Name Prefix \\
\hline Trumpler (1921).... & 3 & $2.5<B<14.5$ & 174 & $\operatorname{Tr}$ \\
\hline Trumpler $(1921)^{\mathrm{a}} \ldots \ldots \ldots \ldots \ldots \ldots \ldots \ldots$ & 24 & $2.5<B<10$ & 72 & $\operatorname{Tr}$ \\
\hline 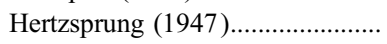 & 4 & $2.5<V<15.5$ & 247 & HII \\
\hline Artyukhina (1969) ...................... & 60 & $2.5<B<12.5$ & $\sim 200$ & $\mathrm{AK}$ \\
\hline Haro et al. (1982) & 20 & $11<V<17.5$ & 519 & HCG \\
\hline van Leeuwen et al. (1986) ........... & 80 & $2.5<B<13$ & 193 & Pels \\
\hline Stauffer et al. (1991) & 16 & $14<V<18$ & 225 & SK \\
\hline Hambly et al. (1993) ....................... & 23 & $10<I<17.5$ & 440 & HHJ \\
\hline Pinfield et al. (2000) ....................... & 6 & $13.5<I<19.5$ & 339 & BPL \\
\hline 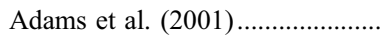 & 300 & $8<K_{s}<14.5$ & 1200 & $\ldots$ \\
\hline Deacon \& Hambly (2004) ............ & 75 & $10<R<19$ & 916 & $\mathrm{DH}$ \\
\hline
\end{tabular}

${ }^{\text {a }}$ The Trumpler paper is listed twice because there are two membership surveys included in that paper, with differing spatial coverages and different limiting magnitudes.

hierarchical triple with three nearly equal mass components). For stars with only photographic optical photometry, where the $1 \sigma$ uncertainties are of order $0.1-0.2 \mathrm{mag}$, we still require the star's photometry to be consistent with membership, but the allowed displacements from the single-star locus are considerably larger. Where accurate radial velocities are known, we require that the star be considered a radial velocity member based on the paper where the radial velocities were presented. Where stars have been previously identified as nonmembers based on photometric or spectroscopic indices, we adopt those conclusions.

Two other relevant pieces of information are sometimes available. In some cases, individual proper-motion membership probabilities are provided by the various membership surveys. If no other information is available, and if the membership probability for a given candidate is less than 0.1 , we exclude that star from our final catalog. However, often a star appears in several catalogs; if it appears in two or more proper-motion membership lists, we include it in the final catalog even if $P<0.1$ in one of those catalogs. Second, an entirely different means to identify candidate Pleiades members is via flare star surveys toward the cluster (Haro et al. 1982; Jones 1981). A star with a formally low membership probability in one catalog but whose photometry is consistent with membership and that was identified as a flare star is retained in our catalog.

Further details of the catalog construction are provided in the Appendix, as are details of the means by which the $B, V$, and $I$ photometry have been homogenized. A full discussion and listing of all of the papers from which we have extracted astrometric and photometric information is also provided in the Appendix. Here we simply provide a very brief description of the inputs to the catalog.

We include candidate cluster members from the following proper-motion surveys: Trumpler (1921), Hertzsprung (1947), Jones (1981), Pels \& Lub (as reported in van Leeuwen et al. 1986), Stauffer et al. (1991), Artyukhina (1969), Hambly et al. (1993), Pinfield et al. (2000), Adams et al. (2001), and Deacon \& Hambly (2004). Another important compilation that provides the initial identification of a significant number of low-mass cluster members is the flare star catalog of Haro et al. (1982). Table 1 provides a brief synopsis of the characteristics of the candidate member catalogs from these papers. The Trumpler paper is listed twice in Table 1 because there are two membership surveys included in that paper, with differing spatial coverages and different limiting magnitudes.
In our final catalog, we have attempted to follow the standard naming convention whereby the primary name is derived from the paper where it was first identified as a cluster member. An exception to this arises for stars with both Trumpler (1921) and Hertzsprung (1947) names, where we use the Hertzsprung numbers as the standard name because that is the most commonly used designation for these stars in the literature. The failure for the Trumpler numbers to be given precedence in the literature perhaps stems from the fact that the Trumpler catalog was published in the Lick Observatory Bulletins as opposed to a refereed journal. In addition to providing a primary name for each star, we provide crossidentifications to some of the other catalogs, particularly where there is existing photometry or spectroscopy of that star using the alternate names. For the brightest cluster members, we provide additional cross-references (e.g., Greek names, Flamsteed numbers, HD numbers).

For each star, we attempt to include an estimate for Johnson $B$ and $V$, and for Cousins $I\left(I_{\mathrm{C}}\right)$. Only a very small fraction of the cluster members have photoelectric photometry in these systems, unfortunately. Photometry for many of the stars has often been obtained in other systems, including Walraven, Geneva, Kron, and Johnson. We have used previously published transformations from the appropriate indices in those systems to Johnson $B V$ or Cousins $I$. In other cases, photometry is available in a natural $I$-band system, primarily for some of the relatively faint cluster members. We have attempted to transform those $I$-band data to $I_{\mathrm{C}}$ by deriving our own conversion using stars for which we already have an $I_{\mathrm{C}}$ estimate as well as the natural $I$ measurement. Details of these issues are provided in the Appendix.

Finally, we have cross-correlated the cluster candidates cata$\log$ with the 2MASS All-Sky PSC and also with the 6x-PSC for the Pleiades. For every star in the catalog, we obtain $J H K_{s}$ photometry and 2MASS positions. Where we have both main survey 2MASS data and data from the $6 \mathrm{x}$ catalog, we adopt the $6 \mathrm{x}$ data for stars with $J>11$, and data from the standard 2MASS catalog otherwise. We verified that the two catalogs do not have any obvious photometric or astrometric offsets relative to each other. The coordinates we list in our catalog are entirely from these 2MASS sources, and hence they inherit the very good and homogeneous 2MASS positional accuracies of order $0.1^{\prime \prime} \mathrm{rms}$.

We have then plotted the candidate Pleiades members in a variety of color-magnitude diagrams and color-color diagrams and required that a star must have photometry that is consistent with cluster membership. Figure 8 illustrates this process and indicates 


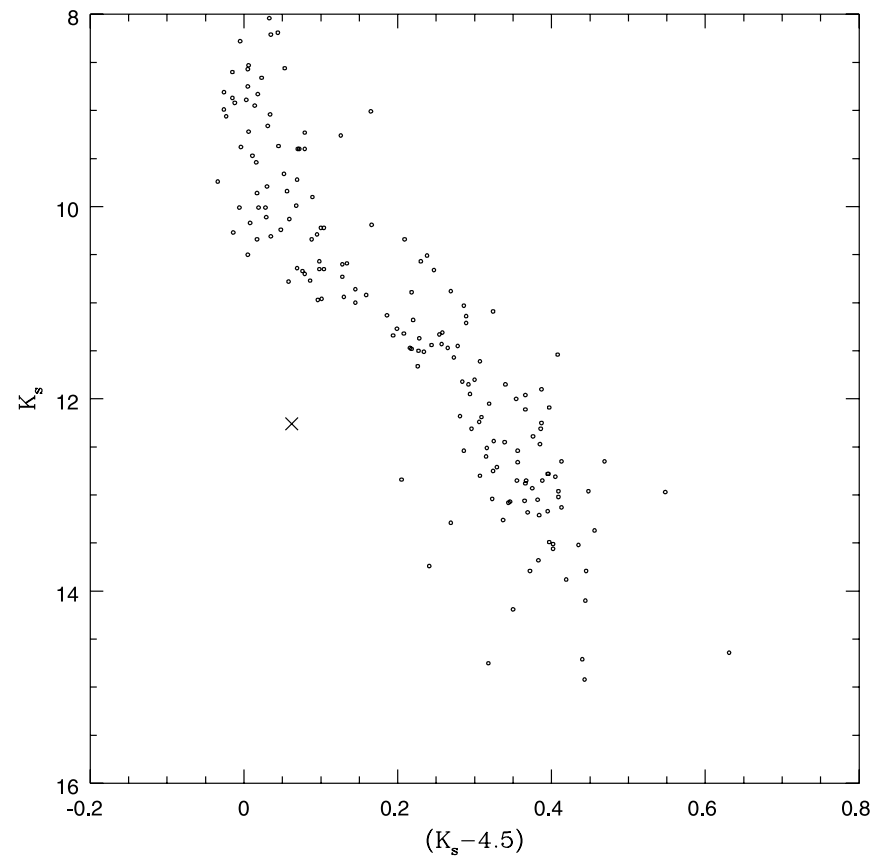

Fig. 8. $-K_{s}$ vs. $K_{s}-[4.5] \mathrm{CMD}$ for Pleiades candidate members, illustrating why we have excluded HII 1695 from the final catalog of cluster members. The "X" symbol marks the location of HII 1695 in this diagram.

why (for example) we have excluded HII 1695 from our final catalog.

Table 2 provides the collected data for the 1417 stars we have retained as candidate Pleiades members. The first two columns are the J2000.0 right ascension and declination from 2MASS; the next are the 2MASS $J H K_{s}$ photometry and their uncertainties, and the 2MASS photometric quality flag ("ph-qual"). If the number following the 2MASS quality flag is a 1, the 2MASS data come from the 2MASS All-Sky PSC; if it is a 2, the data come from the $6 \mathrm{x}-\mathrm{PSC}$. The next three columns provide the $B, V$, and $I_{\mathrm{C}}$ photometry, followed by a flag that indicates the provenance of that photometry. The last column provides the most commonly used names for these stars. The hydrogen-burning mass limit for the Pleiades occurs at about $V=22, I=18, K_{s}=14.4$. Fiftythree of the candidate members in the catalog are fainter than this limit and hence should be substellar if they are indeed Pleiades members.

Table 3 provides the IRAC [3.6], [4.5], [5.8], and [8.0] photometry we have derived for Pleiades candidate members included within the region covered by the IRAC shallow survey of the Pleiades (see $\S 2$ ). The brightest stars are saturated even in our short integration frame data, particularly for the more sensitive 3.6 and $4.5 \mu \mathrm{m}$ channels. At the faint end, we provide photometry only for 3.6 and $4.5 \mu \mathrm{m}$ because the objects are undetected in the two longer wavelength channels. At the "top" and "bottom" of the survey region, we have incomplete wavelength coverage for a band of width about $5^{\prime}$, and for stars in those areas we report only photometry in either the 3.6 and 5.8 bands or the 4.5 and 8.0 bands.

Because Table 2 is an amalgam of many previous catalogs, each of which have different spatial coverage, magnitude limits, and other idiosyncrasies, it is necessarily incomplete and inhomogeneous. It also certainly includes some nonmembers. For $V<12$, we expect very few nonmembers because of the extensive spectroscopic data available for those stars; the fraction of nonmembers will likely increase to fainter magnitudes, particularly for stars located far from the cluster center. The catalog is simply an attempt to collect all of the available data, identify some of the nonmembers, and eliminate duplications. We hope that it will also serve as a starting point for future efforts to produce a "cleaner" catalog.

Figure 9 shows the distribution on the sky of the stars in Table 2. The complete spatial distribution of all members of the Pleiades may differ slightly from what is shown due to the inhomogeneous

TABLE 2

Pleiades Members: Literature Photometry

\begin{tabular}{|c|c|c|c|c|c|c|c|c|c|c|}
\hline $\begin{array}{l}\text { R.A. (J2000.0) } \\
\text { (deg) }\end{array}$ & $\begin{array}{l}\text { Dec. (J2000.0) } \\
\text { (deg) }\end{array}$ & $J$ & $H$ & $K_{s}$ & ph-qual $^{\mathrm{a}}$ & $B$ & V & $I_{\mathrm{C}}$ & Reference & Names \\
\hline $51.898273 \ldots \ldots \ldots \ldots$ & 24.528660 & $10.781 \pm 0.025$ & $10.066 \pm 0.030$ & $9.892 \pm 0.017$ & AAA1 & & & 11.85 & 22 & DH 001 \\
\hline $51.976067 \ldots \ldots \ldots \ldots \ldots$ & 24.936478 & $12.880 \pm 0.019$ & $12.219 \pm 0.030$ & $11.981 \pm 0.016$ & AAA1 & $\ldots$ & $\ldots$ & 14.34 & 22 & DH 003 \\
\hline $52.006481 \ldots \ldots \ldots \ldots$ & 23.078499 & $13.525 \pm 0.022$ & $12.919 \pm 0.022$ & $12.619 \pm 0.021$ & AAA1 & $\ldots$ & $\ldots$ & 15.05 & 22 & DH 004 \\
\hline $52.168613 \ldots \ldots \ldots \ldots . .$. & 25.607782 & $10.198 \pm 0.019$ & $9.883 \pm 0.029$ & $9.723 \pm 0.016$ & AAA1 & 12.59 & 11.75 & $\ldots$ & 9 & AKIII 59 \\
\hline $52.355843 \ldots \ldots \ldots \ldots$ & 25.652304 & $8.459 \pm 0.015$ & $8.314 \pm 0.059$ & $8.270 \pm 0.031$ & AAA1 & 9.90 & 9.43 & & 9 & AKIII 79 \\
\hline $52.409874 \ldots \ldots \ldots \ldots$ & 24.510546 & $10.318 \pm 0.023$ & $9.856 \pm 0.028$ & $9.698 \pm 0.016$ & AAA1 & $\ldots$ & $\ldots$ & 11.19 & 22 & DH 008 \\
\hline $52.494766 \ldots \ldots \ldots \ldots \ldots$ & 23.371859 & $12.799 \pm 0.018$ & $12.206 \pm 0.019$ & $11.946 \pm 0.018$ & AAA1 & $\ldots$ & $\ldots$ & 14.39 & 22 & DH 009 \\
\hline $52.534420 \ldots \ldots \ldots \ldots$ & 22.644163 & $13.680 \pm 0.022$ & $12.968 \pm 0.024$ & $12.770 \pm 0.023$ & AAA1 & $\ldots$ & $\ldots$ & 15.16 & 22 & DH 010 \\
\hline $52.639614 \ldots \ldots \ldots \ldots \ldots$ & 26.215767 & $11.007 \pm 0.018$ & $10.400 \pm 0.027$ & $10.279 \pm 0.016$ & AAA1 & $\ldots$ & $\ldots$ & 11.86 & 22 & DH 011 \\
\hline $52.647411 \ldots \ldots \ldots \ldots$ & 23.052334 & $15.319 \pm 0.051$ & $14.565 \pm 0.060$ & $14.260 \pm 0.073$ & AAA1 & $\ldots$ & $\ldots$ & 16.68 & 22 & DH 012 \\
\hline $52.656086 \ldots \ldots \ldots \ldots$ & 26.346100 & $14.239 \pm 0.026$ & $13.524 \pm 0.037$ & $13.299 \pm 0.032$ & AAA1 & $\ldots$ & $\ldots$ & 15.73 & 22 & DH 013 \\
\hline $53.001957 \ldots \ldots \ldots \ldots . . . .$. & 23.774900 & $11.329 \pm 0.017$ & $10.686 \pm 0.019$ & $10.520 \pm 0.016$ & AAA1 & 15.27 & 13.95 & $\ldots$ & 4 & Pels 109 \\
\hline $53.032749 \ldots \ldots \ldots \ldots$ & 23.232655 & $13.135 \pm 0.019$ & $12.486 \pm 0.019$ & $12.254 \pm 0.018$ & AAA1 & $\ldots$ & $\ldots$ & 14.71 & 22 & DH 017 \\
\hline
\end{tabular}

Noте.-Table 2 is available in its entirety in the electronic edition of the Astrophysical Journal Supplement. A portion is shown here for guidance regarding its form and content.

${ }^{a}$ Standard 2MASS photometric data quality flag for $\mathrm{JHK}_{s}$, in that order. If the number following the 2MASS quality flags is a 1, the 2MASS data come from the standard 2MASS catalog; if it is a 2 , the data come from the deep catalog. 
TABLE 3

Pleiades Members: IRAC Photometry

\begin{tabular}{|c|c|c|c|c|}
\hline Name & {$[3.6]$} & {$[4.5]$} & {$[5.8]$} & {$[8]$} \\
\hline 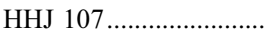 & 12.550 & 12.514 & 12.474 & 12.388 \\
\hline 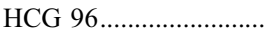 & 11.869 & 11.881 & 11.805 & 11.824 \\
\hline 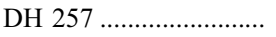 & 9.604 & 9.608 & 9.604 & 9.554 \\
\hline SK $646 \ldots \ldots \ldots \ldots \ldots \ldots \ldots \ldots$ & 11.318 & 11.273 & 11.204 & 11.215 \\
\hline 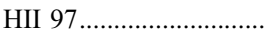 & $\ldots$ & 9.760 & $\ldots$ & 9.666 \\
\hline Pels $056 \ldots \ldots \ldots \ldots \ldots \ldots \ldots \ldots$ & 9.188 & 9.214 & 9.164 & 9.165 \\
\hline HCG $112 \ldots \ldots \ldots \ldots \ldots \ldots \ldots$ & 11.711 & 11.646 & 11.623 & 11.620 \\
\hline 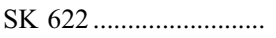 & 11.686 & 11.656 & 11.699 & 11.575 \\
\hline HCG $115 \ldots \ldots \ldots \ldots \ldots \ldots . . . . . . . .$. & 11.450 & 11.434 & 11.316 & 11.437 \\
\hline HII $153 \ldots \ldots \ldots \ldots \ldots \ldots \ldots \ldots$ & 7.163 & 7.205 & 7.183 & 7.198 \\
\hline 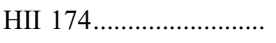 & $\ldots$ & 9.325 & $\ldots$ & 9.285 \\
\hline HII $173 \ldots \ldots \ldots \ldots \ldots \ldots \ldots \ldots$ & 8.798 & 8.812 & 8.763 & 8.768 \\
\hline HCG $125 \ldots \ldots \ldots \ldots \ldots \ldots . . . . . . . .$. & 11.641 & 11.594 & 11.564 & 11.540 \\
\hline Pels $043 \ldots \ldots \ldots \ldots \ldots \ldots \ldots \ldots$ & 9.673 & $\ldots$ & 9.673 & $\ldots$ \\
\hline 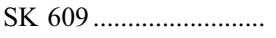 & 15.572 & 15.663 & 15.857 & 15.685 \\
\hline AK 1B146................... & 8.189 & 8.175 & 8.153 & 8.166 \\
\hline 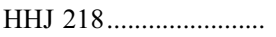 & 12.309 & 12.285 & 12.268 & 12.494 \\
\hline 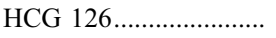 & 11.220 & 11.205 & 11.181 & 11.193 \\
\hline 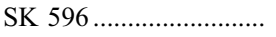 & 11.141 & 11.112 & 11.039 & 11.061 \\
\hline 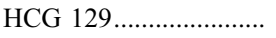 & $\ldots$ & 11.276 & $\ldots$ & 11.282 \\
\hline 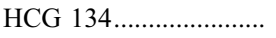 & 11.111 & 11.071 & 11.032 & 11.033 \\
\hline 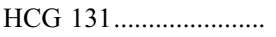 & 9.941 & 9.982 & 9.980 & 9.911 \\
\hline 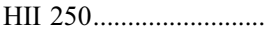 & $\ldots$ & 9.083 & $\ldots$ & 9.023 \\
\hline 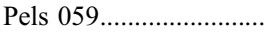 & 9.950 & 9.991 & 9.951 & 9.934 \\
\hline 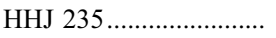 & 12.167 & 12.085 & 11.940 & 12.080 \\
\hline 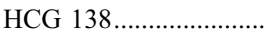 & 11.200 & 11.132 & 11.096 & 11.124 \\
\hline 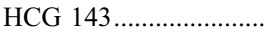 & 11.355 & 11.303 & 11.258 & 11.291 \\
\hline 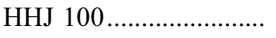 & 12.588 & 12.551 & 12.462 & 12.459 \\
\hline HCG $152 \ldots \ldots \ldots \ldots \ldots \ldots \ldots$ & 10.858 & 10.874 & 10.857 & 10.846 \\
\hline 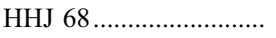 & 13.007 & 12.914 & 13.089 & 12.688 \\
\hline 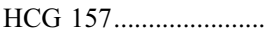 & 12.194 & $\ldots$ & 12.045 & $\ldots$ \\
\hline 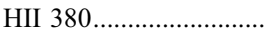 & 10.169 & 10.192 & 10.173 & 10.161 \\
\hline 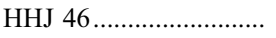 & 13.149 & 13.085 & 13.170 & 12.898 \\
\hline 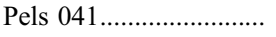 & 9.740 & 9.774 & 9.674 & 9.698 \\
\hline 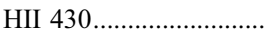 & 9.509 & 9.459 & 9.356 & 9.465 \\
\hline 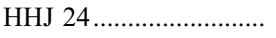 & 13.410 & 13.345 & 13.298 & 13.784 \\
\hline 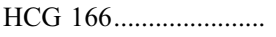 & 12.168 & 12.111 & 11.908 & 12.291 \\
\hline 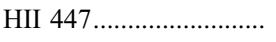 & $\ldots$ & $\ldots$ & 5.522 & 5.528 \\
\hline HII $468 \ldots \ldots \ldots \ldots \ldots \ldots \ldots$ & $\ldots$ & $\ldots$ & 4.010 & 3.910 \\
\hline 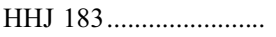 & 12.458 & 12.426 & 12.431 & 12.207 \\
\hline HII $489 \ldots \ldots \ldots \ldots \ldots \ldots \ldots \ldots$ & 8.857 & 8.885 & 8.864 & 8.814 \\
\hline DH 367 ....................... & 12.770 & 12.717 & 12.594 & 12.589 \\
\hline 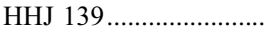 & $\ldots$ & 12.495 & $\ldots$ & 12.628 \\
\hline 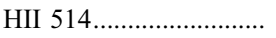 & 9.019 & 9.006 & 8.955 & 8.978 \\
\hline 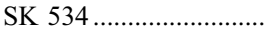 & 11.269 & 11.262 & 11.163 & 11.241 \\
\hline 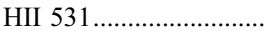 & 7.715 & 7.731 & 7.703 & 7.719 \\
\hline 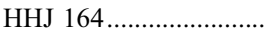 & 12.469 & 12.381 & 12.354 & 12.410 \\
\hline 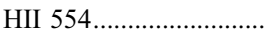 & $\ldots$ & 10.456 & $\ldots$ & 10.427 \\
\hline 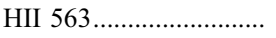 & $\ldots$ & $\ldots$ & 4.620 & 4.580 \\
\hline 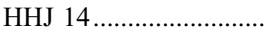 & 13.479 & 13.461 & 13.624 & 13.430 \\
\hline 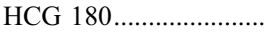 & 12.854 & 12.775 & 12.763 & 12.697 \\
\hline 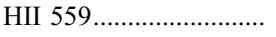 & $\ldots$ & 10.120 & $\ldots$ & 10.097 \\
\hline 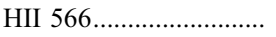 & $\ldots$ & 10.722 & $\ldots$ & 10.681 \\
\hline 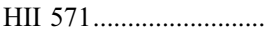 & 9.175 & 9.151 & 9.161 & 9.097 \\
\hline 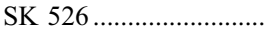 & $\ldots$ & 10.744 & $\ldots$ & 10.698 \\
\hline 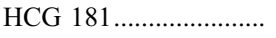 & $\ldots$ & 11.173 & $\ldots$ & 11.141 \\
\hline 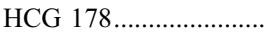 & 10.938 & 10.960 & 10.774 & 10.767 \\
\hline 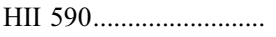 & $\ldots$ & 10.552 & $\ldots$ & 10.503 \\
\hline 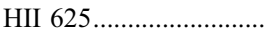 & 9.317 & 9.330 & 9.323 & 9.250 \\
\hline 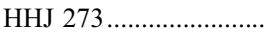 & $\ldots$ & 12.014 & $\ldots$ & 11.962 \\
\hline 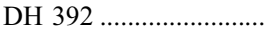 & 11.972 & 11.934 & 11.827 & 12.053 \\
\hline 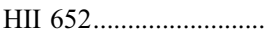 & 7.426 & 7.455 & 7.422 & 7.278 \\
\hline 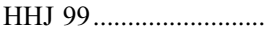 & 13.052 & 13.021 & 13.180 & 13.126 \\
\hline 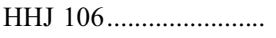 & 12.975 & 12.923 & 12.953 & 12.619 \\
\hline HII $676 \ldots \ldots \ldots \ldots \ldots \ldots \ldots \ldots$ & 10.149 & 10.131 & 10.105 & 10.132 \\
\hline
\end{tabular}

TABLE 3-Continued

\begin{tabular}{|c|c|c|c|c|}
\hline Name & {$[3.6]$} & {$[4.5]$} & {$[5.8]$} & {$[8]$} \\
\hline HII $673 \ldots \ldots \ldots . . . .$. & 10.938 & 10.944 & 10.910 & 10.997 \\
\hline HHJ -293................... & 12.227 & $\ldots$ & 12.060 & \\
\hline HII $686 \ldots \ldots \ldots \ldots$ & 10.100 & 10.116 & 10.126 & 10.084 \\
\hline 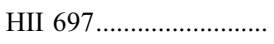 & 7.703 & 7.661 & 7.656 & 7.597 \\
\hline 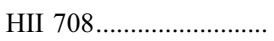 & 8.547 & 8.507 & 8.472 & 8.497 \\
\hline HII 717........................ & 6.538 & 6.600 & 6.592 & 6.611 \\
\hline HCG 196...................... & 10.767 & 10.810 & 10.767 & 10.749 \\
\hline HHJ $130 \ldots \ldots \ldots \ldots \ldots \ldots$ & 12.724 & 12.695 & 12.618 & 12.781 \\
\hline HII 738............................ & 8.819 & 8.845 & 8.773 & 8.762 \\
\hline HII $745 \ldots \ldots \ldots \ldots \ldots \ldots$ & 8.002 & 8.007 & 7.975 & 7.988 \\
\hline HCG 195 .................... & 10.648 & 10.611 & 10.561 & 10.587 \\
\hline HII 746......................... & 9.300 & 9.328 & 9.292 & 9.280 \\
\hline HII 740.......................... & $\ldots$ & 10.495 & $\ldots$ & 10.425 \\
\hline HII $762 \ldots \ldots \ldots \ldots \ldots$ & 10.556 & 10.571 & 10.533 & 10.479 \\
\hline 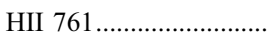 & 8.736 & 8.745 & 8.722 & 8.694 \\
\hline 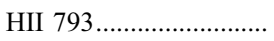 & 10.529 & 10.621 & 10.493 & 10.431 \\
\hline DH 403 & 14.486 & 14.432 & 14.604 & 13.951 \\
\hline BPL $77 \ldots \ldots \ldots \ldots \ldots$ & 11.528 & 11.500 & 11.390 & 55.505 \\
\hline 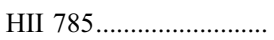 & $\ldots$ & $\ldots$ & 4.050 & 4.030 \\
\hline HII 799 & 10.140 & 10.162 & 10.107 & 10.178 \\
\hline 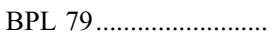 & 14.069 & 14.009 & 13.914 & 13.960 \\
\hline 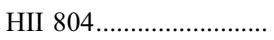 & 7.345 & 7.323 & 7.340 & 7.338 \\
\hline HHJ $166 \ldots \ldots \ldots \ldots$ & 12.469 & 12.405 & 12.364 & 12.410 \\
\hline BPL $81 \ldots \ldots$ & 14.498 & 14.477 & 14.190 & 14.098 \\
\hline HII $813 \ldots \ldots \ldots \ldots \ldots \ldots . . .$. & 10.355 & 10.340 & 10.283 & 10.288 \\
\hline HII $817 \ldots \ldots \ldots \ldots \ldots \ldots$ & $\ldots$ & 9.900 & $\ldots$ & 5.737 \\
\hline BPL $82 \ldots \ldots \ldots$ & 11.539 & 11.510 & 11.449 & 11.582 \\
\hline SK $497 \ldots \ldots$ & 11.198 & 11.172 & 11.112 & 11.107 \\
\hline HHJ $27 \ldots \ldots \ldots \ldots \ldots$ & 13.407 & 13.418 & 13.113 & 13.243 \\
\hline DH 412 & 13.309 & 13.297 & 13.161 & 13.409 \\
\hline HHJ $127 \ldots \ldots \ldots \ldots \ldots$ & 12.785 & 12.736 & 12.774 & 12.486 \\
\hline SK 491 & 11.042 & 11.052 & 10.994 & 11.016 \\
\hline HII $870 \ldots \ldots \ldots \ldots$ & 9.133 & 9.134 & 9.109 & 9.082 \\
\hline 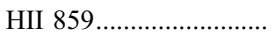 & $\ldots$ & 6.454 & $\ldots$ & 6.422 \\
\hline SK $490 \ldots \ldots \ldots \ldots$ & 10.656 & 10.684 & 10.635 & 10.672 \\
\hline HHJ 363 & 11.628 & 11.536 & 11.505 & 11.552 \\
\hline BPL $88 \ldots \ldots \ldots \ldots$ & 12.871 & 12.811 & 12.703 & 12.560 \\
\hline SK 488 & $\ldots$ & 11.196 & $\ldots$ & 11.137 \\
\hline 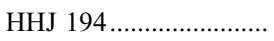 & 12.575 & 12.635 & 12.546 & 12.712 \\
\hline 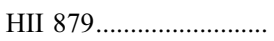 & $\ldots$ & 10.081 & $\ldots$ & 10.066 \\
\hline HHJ 435 & 10.767 & 10.761 & 10.733 & 10.702 \\
\hline HII 883 & $\ldots$ & 10.195 & $\ldots$ & 10.125 \\
\hline HII $890 \ldots \ldots \ldots \ldots . .$. & 10.727 & 10.715 & 10.649 & 10.696 \\
\hline HII 916 ............... & $\ldots$ & 9.524 & $\ldots$ & 9.479 \\
\hline HII 930 & 10.459 & 10.472 & 10.424 & 10.459 \\
\hline 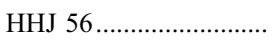 & 12.475 & 12.422 & 12.572 & 12.562 \\
\hline 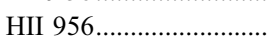 & 7.092 & 7.131 & 7.079 & 7.069 \\
\hline HII 980.......................... & $\ldots$ & $\ldots$ & $\ldots$ & 4.190 \\
\hline HHJ $105 \ldots \ldots \ldots \ldots$ & $\ldots$ & 12.724 & $\ldots$ & 12.609 \\
\hline DH 441 & 11.766 & 11.693 & 11.531 & 11.636 \\
\hline 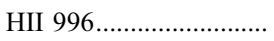 & 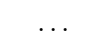 & 8.932 & 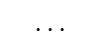 & 8.878 \\
\hline 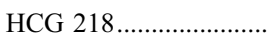 & 12.534 & 12.462 & 12.443 & 12.465 \\
\hline HHJ $249 \ldots \ldots \ldots \ldots \ldots \ldots$ & 12.259 & 12.254 & 12.173 & 12.154 \\
\hline HCG 219.................... & 10.889 & 10.851 & 10.794 & 10.801 \\
\hline HHJ $326 \ldots \ldots \ldots \ldots \ldots \ldots$ & 11.786 & 11.731 & 11.618 & 11.719 \\
\hline HII $1028 \ldots \ldots \ldots \ldots$ & 7.078 & 7.120 & 7.113 & 7.085 \\
\hline HII $1015 \ldots \ldots \ldots \ldots \ldots \ldots \ldots$ & & 9.016 & & 8.965 \\
\hline HHJ $161 \ldots \ldots \ldots \ldots \ldots \ldots$ & 12.285 & 12.181 & 12.275 & 12.181 \\
\hline HII $1039 \ldots \ldots \ldots \ldots \ldots \ldots \ldots$ & 9.798 & $\ldots$ & 9.764 & $\ldots$ \\
\hline HII $1032 \ldots \ldots \ldots \ldots \ldots \ldots \ldots$ & 9.143 & 9.129 & 9.144 & 9.071 \\
\hline HII $1061 \ldots \ldots \ldots \ldots$ & 10.298 & 10.323 & 10.245 & 10.240 \\
\hline 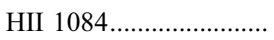 & 7.052 & & 7.043 & \\
\hline 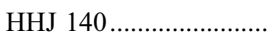 & 12.439 & 12.385 & 12.431 & 12.409 \\
\hline 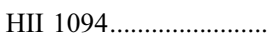 & 10.549 & 10.546 & 10.479 & 10.613 \\
\hline 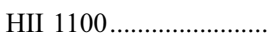 & 9.285 & 9.321 & 9.302 & 9.264 \\
\hline HII $1117 \ldots \ldots \ldots \ldots \ldots \ldots$ & 8.497 & 8.524 & 8.543 & 8.482 \\
\hline
\end{tabular}


TABLE 3-Continued

\begin{tabular}{|c|c|c|c|c|}
\hline Name & [3.6] & {$[4.5]$} & {$[5.8]$} & [8] \\
\hline HII $1110 \ldots \ldots \ldots \ldots \ldots \ldots$ & 10.227 & 10.284 & 10.240 & 10.208 \\
\hline HII $1122 \ldots \ldots \ldots \ldots \ldots$. & 8.149 & 8.146 & 8.174 & 8.132 \\
\hline HII $1124 \ldots \ldots \ldots \ldots \ldots \ldots \ldots . . .$. & 9.858 & 9.843 & 9.847 & 9.785 \\
\hline HHJ 104 ........................ & 12.705 & $\ldots$ & 12.752 & $\ldots$ \\
\hline DH 467 & 11.379 & 11.297 & 11.253 & 11.271 \\
\hline HII $1173 \ldots \ldots \ldots \ldots \ldots \ldots \ldots$ & & 10.855 & $\ldots$ & 10.781 \\
\hline HHJ $247 \ldots \ldots \ldots \ldots \ldots . . .$. & 11.836 & & 11.660 & \\
\hline HCG 244....................... & 10.955 & 10.921 & 10.873 & 10.870 \\
\hline HII $1215 \ldots \ldots \ldots \ldots \ldots \ldots \ldots \ldots$ & 8.997 & $\ldots$ & 8.953 & $\ldots$ \\
\hline HHJ $257 \ldots \ldots \ldots \ldots \ldots \ldots \ldots$ & 11.948 & 11.924 & 11.906 & 11.714 \\
\hline HHJ 174 & 12.506 & $\ldots$ & $\ldots$ & $\ldots$ \\
\hline HHJ $299 \ldots \ldots \ldots \ldots \ldots \ldots \ldots$ & 11.797 & 11.744 & 11.694 & 11.663 \\
\hline HII $1234 \ldots \ldots \ldots \ldots \ldots \ldots \ldots$ & 6.729 & 6.743 & 6.712 & 6.679 \\
\hline 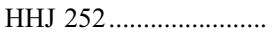 & 11.927 & 11.863 & 11.740 & 11.836 \\
\hline 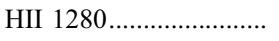 & 10.548 & 10.602 & 10.528 & 10.575 \\
\hline HII 1286 ......................... & 10.378 & $\ldots$ & 10.289 & $\ldots$ \\
\hline HII $1284 \ldots \ldots \ldots \ldots \ldots \ldots . . .$. & 7.617 & 7.627 & 7.571 & 7.589 \\
\hline HII 1298 ....................... & 9.778 & 9.784 & 9.800 & 9.741 \\
\hline HCG $253 \ldots \ldots \ldots \ldots \ldots \ldots$ & 11.625 & 11.513 & 11.511 & 11.559 \\
\hline HII 1306 ......................... & 9.798 & 9.811 & 9.773 & 9.747 \\
\hline HHJ 37 ......................... & 13.241 & 13.158 & 13.051 & 13.073 \\
\hline HII $1321 \ldots \ldots \ldots \ldots \ldots \ldots \ldots . .$. & 10.438 & 10.413 & 10.361 & 10.372 \\
\hline HII 1309.................... & 8.270 & 8.285 & 8.244 & 8.259 \\
\hline HHJ 92 ......................... & 12.816 & 12.717 & 12.684 & 12.753 \\
\hline HII $1332 \ldots \ldots \ldots \ldots \ldots \ldots \ldots$ & 9.969 & 10.016 & 9.991 & 9.958 \\
\hline HCG 258....................... & 10.839 & 10.766 & 10.673 & 10.750 \\
\hline HII 1338 ......................... & 7.463 & 7.483 & 7.503 & 7.476 \\
\hline HII 1348 ......................... & 9.622 & 9.651 & 9.622 & 9.575 \\
\hline HII $1355 \ldots \ldots \ldots \ldots \ldots \ldots . . .$. & 10.016 & 10.024 & 9.991 & 10.035 \\
\hline HII $1362 \ldots \ldots \ldots \ldots \ldots \ldots . . .$. & 7.637 & 7.661 & 7.641 & 7.618 \\
\hline HII $1380 \ldots \ldots \ldots \ldots \ldots \ldots . .$. & 6.914 & 6.952 & 6.961 & 6.962 \\
\hline 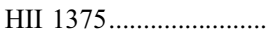 & $\ldots$ & 6.323 & 6.311 & 6.318 \\
\hline HCG 266........................ & 12.175 & 12.115 & 12.078 & 11.993 \\
\hline HII $1384 \ldots \ldots \ldots \ldots \ldots \ldots \ldots$ & $\ldots$ & 6.984 & $\ldots$ & 6.979 \\
\hline HII 1397........................ & 7.181 & 7.196 & 7.192 & 7.184 \\
\hline HHJ $198 \ldots \ldots \ldots \ldots \ldots \ldots \ldots$ & 12.717 & 12.668 & 12.541 & 12.431 \\
\hline HCG 269...................... & 11.965 & $\ldots$ & 11.859 & $\ldots$ \\
\hline HII $1425 \ldots \ldots \ldots \ldots \ldots \ldots \ldots$ & 7.342 & $\ldots$ & 7.323 & $\ldots$ \\
\hline HII 1431 ......................... & 6.640 & 6.652 & 6.644 & 6.674 \\
\hline HCG 273 ..................... & 11.175 & 11.142 & 11.093 & 11.240 \\
\hline HCG 277...................... & 10.658 & & 10.553 & \\
\hline 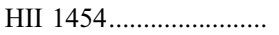 & $\ldots$ & 10.071 & $\ldots$ & 10.026 \\
\hline DH 523 & 12.495 & 12.493 & 12.472 & 12.348 \\
\hline HII 1516 ......................... & 10.219 & 10.252 & 10.211 & 10.217 \\
\hline 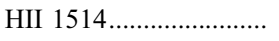 & 8.940 & 8.936 & 8.910 & 8.913 \\
\hline HII $1532 \ldots \ldots \ldots \ldots \ldots \ldots \ldots$ & 10.510 & 10.472 & 10.450 & 10.403 \\
\hline HII $1531 \ldots \ldots \ldots \ldots \ldots \ldots . .$. & 10.260 & 10.275 & 10.191 & 10.195 \\
\hline HHJ 26........................ & 13.951 & 13.840 & 13.870 & 13.714 \\
\hline 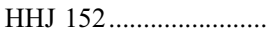 & 12.384 & $\ldots$ & 12.228 & $\ldots$ \\
\hline 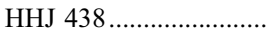 & 11.138 & 11.076 & 11.014 & 11.048 \\
\hline HCG 295 ........................ & 11.248 & 11.254 & 11.248 & 11.190 \\
\hline 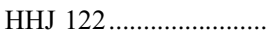 & 12.698 & & 12.635 & \\
\hline HII $1613 \ldots \ldots \ldots \ldots \ldots \ldots . .$. & 8.562 & 8.565 & 8.514 & 8.535 \\
\hline HHJ $240 \ldots \ldots \ldots \ldots . . . .$. & 12.232 & 12.184 & 12.131 & 12.102 \\
\hline HII 1726 ......................... & 7.905 & 7.904 & 7.883 & 7.891 \\
\hline HCG 307......................... & 11.929 & 11.899 & 11.830 & 11.887 \\
\hline HHJ $156 \ldots \ldots \ldots \ldots \ldots \ldots$ & 12.458 & & 12.384 & \\
\hline HHJ $225 \ldots \ldots \ldots \ldots \ldots \ldots$ & 12.370 & 12.237 & 12.267 & 12.356 \\
\hline DH 555 & 13.164 & 13.108 & 12.971 & 13.066 \\
\hline HCG $311 \ldots \ldots \ldots \ldots \ldots \ldots$ & 12.095 & 12.014 & 11.969 & 12.067 \\
\hline HII $1762 \ldots \ldots \ldots \ldots \ldots \ldots$ & 7.306 & 7.312 & 7.342 & 7.332 \\
\hline HCG $315 \ldots \ldots \ldots \ldots \ldots \ldots$ & 11.888 & $\ldots$ & 11.717 & $\ldots$ \\
\hline 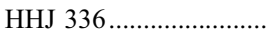 & 11.528 & & 11.462 & \\
\hline HII 1797 ....................... & 8.729 & & 8.662 & \\
\hline 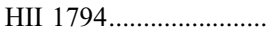 & 8.871 & 8.887 & 8.812 & 8.797 \\
\hline HII $1785 \ldots \ldots \ldots \ldots \ldots \ldots \ldots$ & 10.569 & 10.594 & 10.552 & 10.563 \\
\hline
\end{tabular}

TABLE 3-Continued

\begin{tabular}{|c|c|c|c|c|}
\hline Name & [3.6] & [4.5] & {$[5.8]$} & [8] \\
\hline НHJ $188 \ldots$. & 12.467 & 12.384 & 12.441 & 12.456 \\
\hline HII 1827 ................. & 10.298 & 10.272 & 10.163 & 10.217 \\
\hline 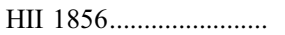 & 8.648 & 8.637 & 8.613 & 8.626 \\
\hline 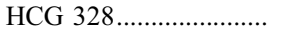 & 12.677 & 12.611 & 12.659 & 12.887 \\
\hline 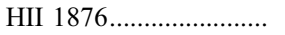 & 6.575 & 6.578 & 6.595 & 6.592 \\
\hline 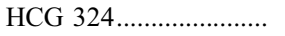 & 11.169 & 11.146 & 11.114 & 11.146 \\
\hline 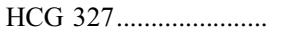 & 12.521 & 12.483 & 12.455 & 12.506 \\
\hline 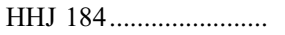 & 12.586 & 12.555 & 12.573 & 12.553 \\
\hline HII $1912 \ldots \ldots \ldots \ldots \ldots \ldots \ldots$ & 7.798 & 7.834 & 7.783 & 7.790 \\
\hline HCG $335 \ldots \ldots \ldots \ldots \ldots \ldots \ldots . . .$. & 12.866 & 12.826 & 12.760 & 12.820 \\
\hline HCG $337 \ldots \ldots \ldots \ldots \ldots \ldots \ldots . . . .$. & 11.627 & 11.558 & 11.584 & 11.665 \\
\hline 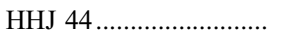 & 13.179 & 13.093 & 13.023 & 12.903 \\
\hline 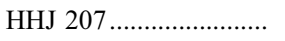 & 12.389 & 12.304 & 12.221 & 12.330 \\
\hline HII 2027 ......................... & 8.788 & 8.836 & 8.774 & 8.784 \\
\hline 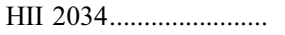 & 9.878 & 9.922 & 9.923 & 9.865 \\
\hline DH 593 & 14.247 & 14.270 & 14.972 & 14.808 \\
\hline 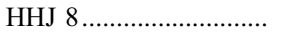 & 13.674 & 13.656 & 13.473 & 13.729 \\
\hline 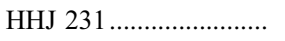 & 12.236 & 12.194 & 12.171 & 12.189 \\
\hline 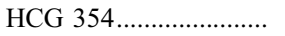 & 10.566 & $\ldots$ & 10.486 & $\ldots$ \\
\hline 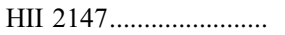 & 8.558 & 8.615 & 8.514 & 8.549 \\
\hline HII 2168........................ & $\ldots$ & $\ldots$ & 3.840 & 3.820 \\
\hline HII $2195 \ldots \ldots \ldots \ldots \ldots \ldots \ldots \ldots . . .$. & 7.600 & 7.622 & 7.601 & 7.588 \\
\hline DH 610 & 13.173 & & 13.132 & \\
\hline HII $2284 \ldots \ldots \ldots \ldots \ldots . .$. & 9.366 & 9.384 & 9.353 & 9.319 \\
\hline HII $2311 \ldots \ldots \ldots \ldots \ldots \ldots$ & 9.445 & $\ldots$ & 9.395 & $\ldots$ \\
\hline HHJ $142 \ldots \ldots \ldots \ldots \ldots \ldots \ldots$ & 12.426 & $\ldots$ & 12.224 & $\ldots$ \\
\hline
\end{tabular}

properties of the proper-motion surveys. However, we believe that those effects are relatively small and the distribution shown is mostly representative of the parent population. One thing that is evident in Figure 9 is mass segregation - the highest mass cluster members are much more centrally located than the lowest mass cluster members. This fact is reinforced by calculating the cumulative number of stars as a function of distance from the cluster center for different absolute magnitude bins. Figure 10 illustrates this fact. Another property of the Pleiades illustrated by Figure 10 is that the cluster appears to be elongated parallel to the Galactic plane, as expected from $n$-body simulations of galactic clusters (Terlevich 1987). Similar plots showing the flattening of the

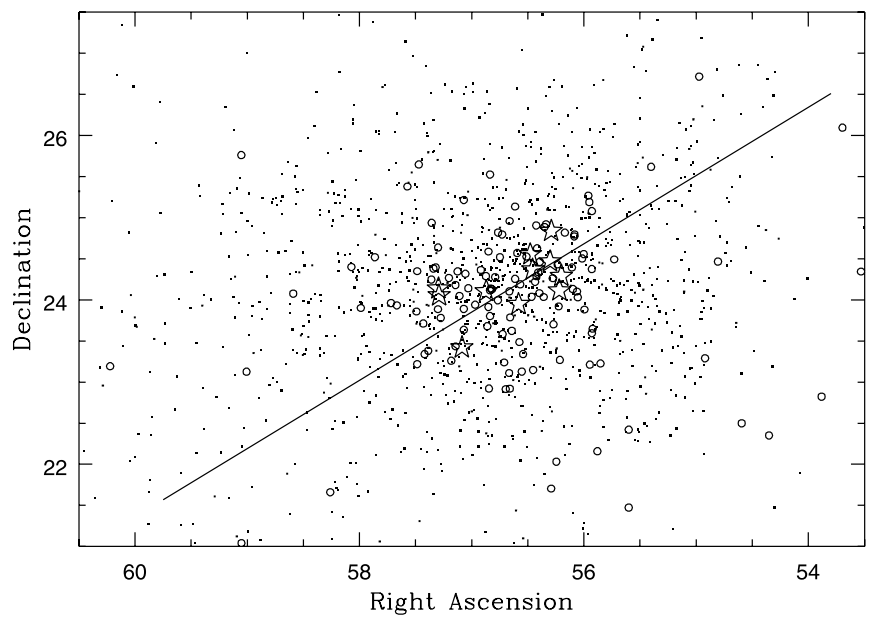

FIG. 9.- Spatial plot of the candidate Pleiades members from Table 2. The large star symbols are members brighter than $K_{s}=6$; the open circles are stars with $6<K_{s}<9$; and the dots are candidate members fainter than $K_{s}=9$. The solid line is parallel to the Galactic plane. 


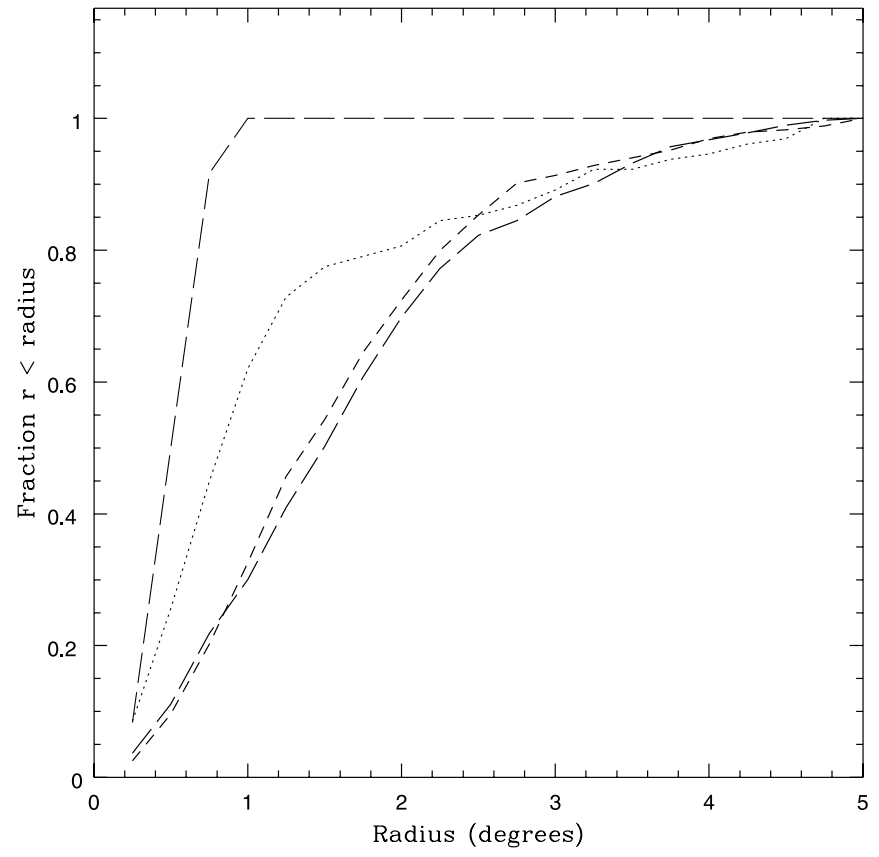

Fig. 10.-Cumulative radial density profiles for Pleiades members in several magnitude ranges: heavy, long-dashed line, $K_{s}<6$; dots, $6<K_{s}<9$; short-dashed line, $9<K_{s}<12$; light, long-dashed line, $K_{s}>12$.

cluster and evidence for mass segregation for the $V<12$ cluster members were provided by Raboud \& Mermilliod (1998).

\section{EMPIRICAL PLEIADES ISOCHRONES AND COMPARISON TO MODEL ISOCHRONES}

Young, nearby, rich open clusters like the Pleiades can and should be used to provide template data that can help interpret observations of more distant clusters or to test theoretical models. The identification of candidate members of distant open clusters is

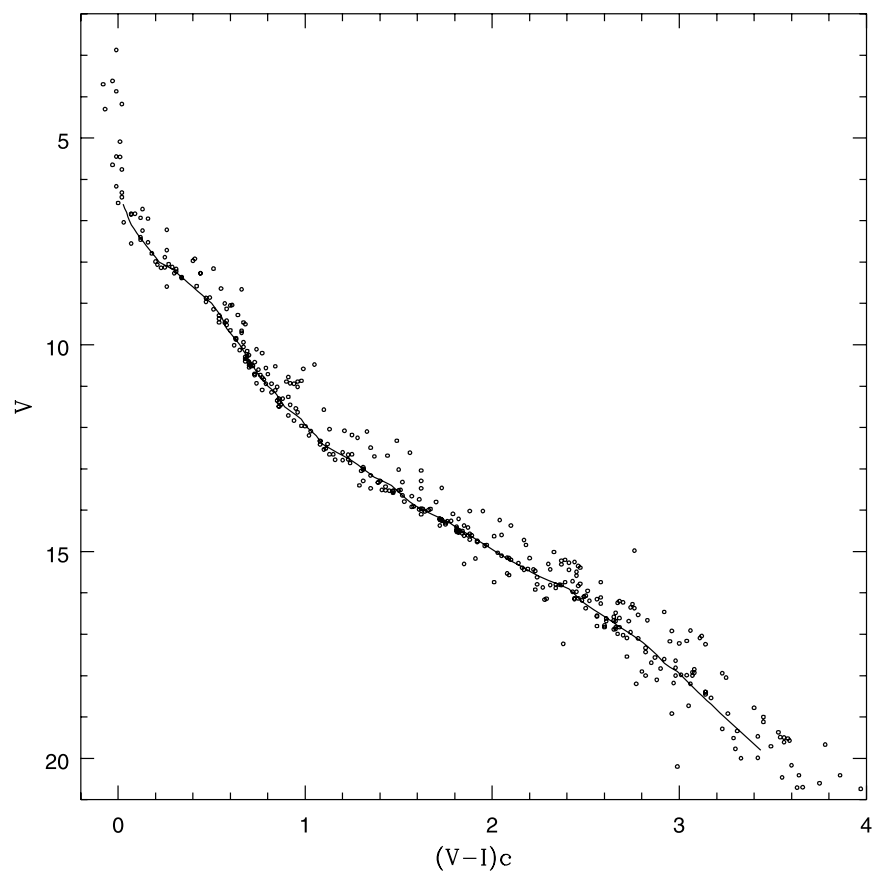

FIG. 11. $-V$ vs. $(V-I)$ CMD for Pleiades members with photoelectric photometry. The solid curve is the "by-eye" fit to the single-star locus for Pleiades members.

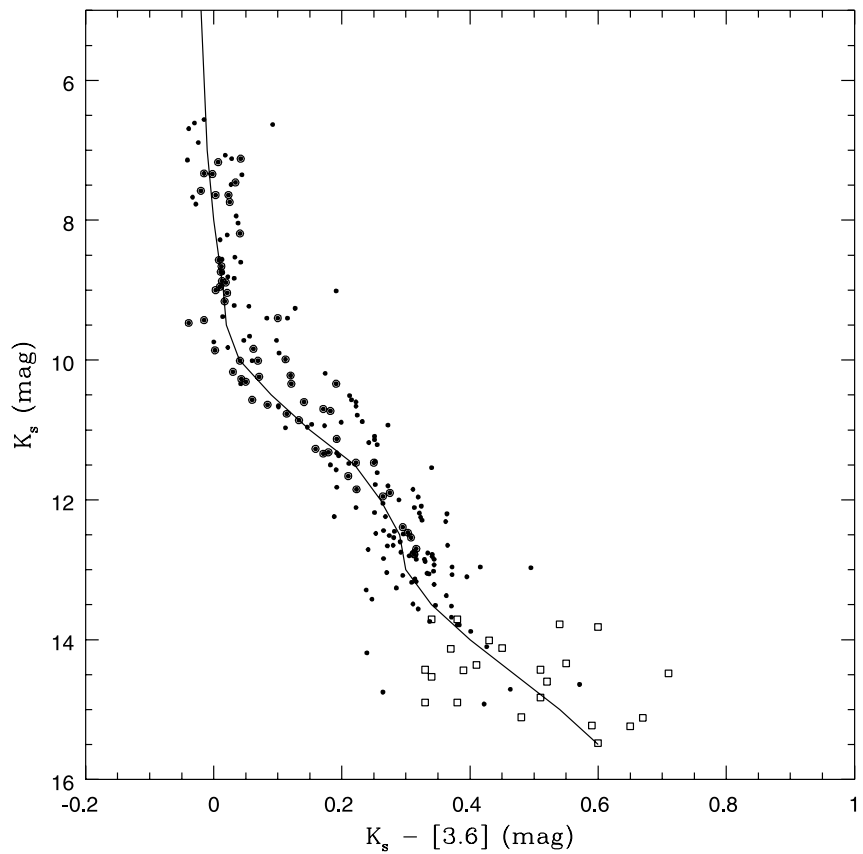

FIG. 12. $-K_{s}$ vs. $K_{s}-[3.6]$ CMD for Pleiades candidate members from Table 2 (dots) and from deeper imaging of a set of Pleiades VLM and brown dwarf candidate members from P. Lowrance et al. (2007, in preparation) (squares). The solid curve is the single-star locus from Table 4.

often based on plots of stars in a color-magnitude diagram, overlaid on which is a line meant to define the single-star locus at the distance of the cluster. The stars lying near or slightly above the locus are chosen as possible or probable cluster members. The data we have collected for the Pleiades provide a means to define the single-star locus for $100 \mathrm{Myr}$, solar metallicity stars in a variety of widely used color systems down to and slightly below the hydrogen-burning mass limit. Figures 11 and 12 illustrate the

TABLE 4

Single-Star Pleiades Loci

\begin{tabular}{|c|c|c|c|c|c|c|c|}
\hline$B$ & V & $I_{\mathrm{C}}$ & $K_{s}$ & [3.6] & {$[4.5]$} & [5.8] & [8] \\
\hline $6.598 \ldots \ldots \ldots$ & 6.600 & 6.574 & 6.592 & 6.602 & 6.615 & 6.602 & 6.602 \\
\hline $6.706 \ldots \ldots \ldots \ldots$ & 6.700 & 6.665 & 6.671 & 6.682 & 6.695 & 6.682 & 6.682 \\
\hline $6.814 \ldots \ldots \ldots \ldots$ & 6.800 & 6.755 & 6.750 & 6.761 & 6.775 & 6.762 & 6.762 \\
\hline $6.922 \ldots$. & 6.900 & 6.848 & 6.834 & 6.841 & 6.855 & 6.841 & 6.841 \\
\hline $7.030 \ldots$ & 7.000 & 6.940 & 6.910 & 6.920 & 6.935 & 6.921 & 6.921 \\
\hline $7.142 \ldots \ldots \ldots \ldots$ & 7.100 & 7.030 & 6.982 & 6.990 & 7.005 & 6.991 & 6.991 \\
\hline $7.254 \ldots$ & 7.200 & 7.115 & 7.039 & 7.050 & 7.064 & 7.050 & 7.049 \\
\hline $7.370 \ldots$ & 7.300 & 7.200 & 7.104 & 7.109 & 7.124 & 7.108 & 7.107 \\
\hline $7.490 \ldots$ & 7.400 & 7.283 & 7.162 & 7.168 & 7.183 & 7.165 & 7.164 \\
\hline $7.610 \ldots$ & 7.500 & 7.367 & 7.228 & 7.238 & 7.252 & 7.233 & 7.231 \\
\hline $7.730 \ldots$. & 7.600 & 7.450 & 7.287 & 7.297 & 7.311 & 7.291 & 7.288 \\
\hline $7.850 \ldots$ & 7.700 & 7.533 & 7.345 & 7.347 & 7.360 & 7.339 & 7.336 \\
\hline $7.968 \ldots$ & 7.800 & 7.615 & 7.387 & .396 & 7.409 & 7.387 & 7.384 \\
\hline $8.084 \ldots$ & 7.900 & 7.698 & 7.428 & & 7.449 & 7.426 & 7.422 \\
\hline $8.200 \ldots \ldots \ldots$ & 8.000 & 7.780 & 7.469 & 7.475 & & 7.465 & 7.460 \\
\hline $8.320 \ldots \ldots \ldots$ & 8.100 & 7.840 & 7.508 & 7.515 & 7.527 & 7.503 & 7.498 \\
\hline $8.440 \ldots \ldots \ldots \ldots . .$. & 8.200 & 7.900 & 7.546 & 7.555 & 7.567 & 7.542 & 7.536 \\
\hline $8.564 \ldots \ldots \ldots \ldots . .$. & 8.300 & 7.975 & 7.591 & 7.594 & 7.606 & 7.580 & 7.575 \\
\hline $8.692 \ldots \ldots \ldots \ldots$ & 8.400 & 8.050 & 7.648 & 7.654 & 7.665 & 7.638 & 7.632 \\
\hline $8.820 \ldots \ldots \ldots \ldots$ & 8.500 & 8.125 & 7.701 & 7.703 & 7.715 & 7.687 & 7.680 \\
\hline $8.936 \ldots \ldots \ldots . .$. & 8.600 & 8.200 & 7.762 & 7.762 & 7.774 & 7.744 & 7.737 \\
\hline
\end{tabular}

Noте.-Table 4 is available in its entirety in the electronic edition of the $A s$ trophysical Journal Supplement. A portion is shown here for guidance regarding its form and content. 

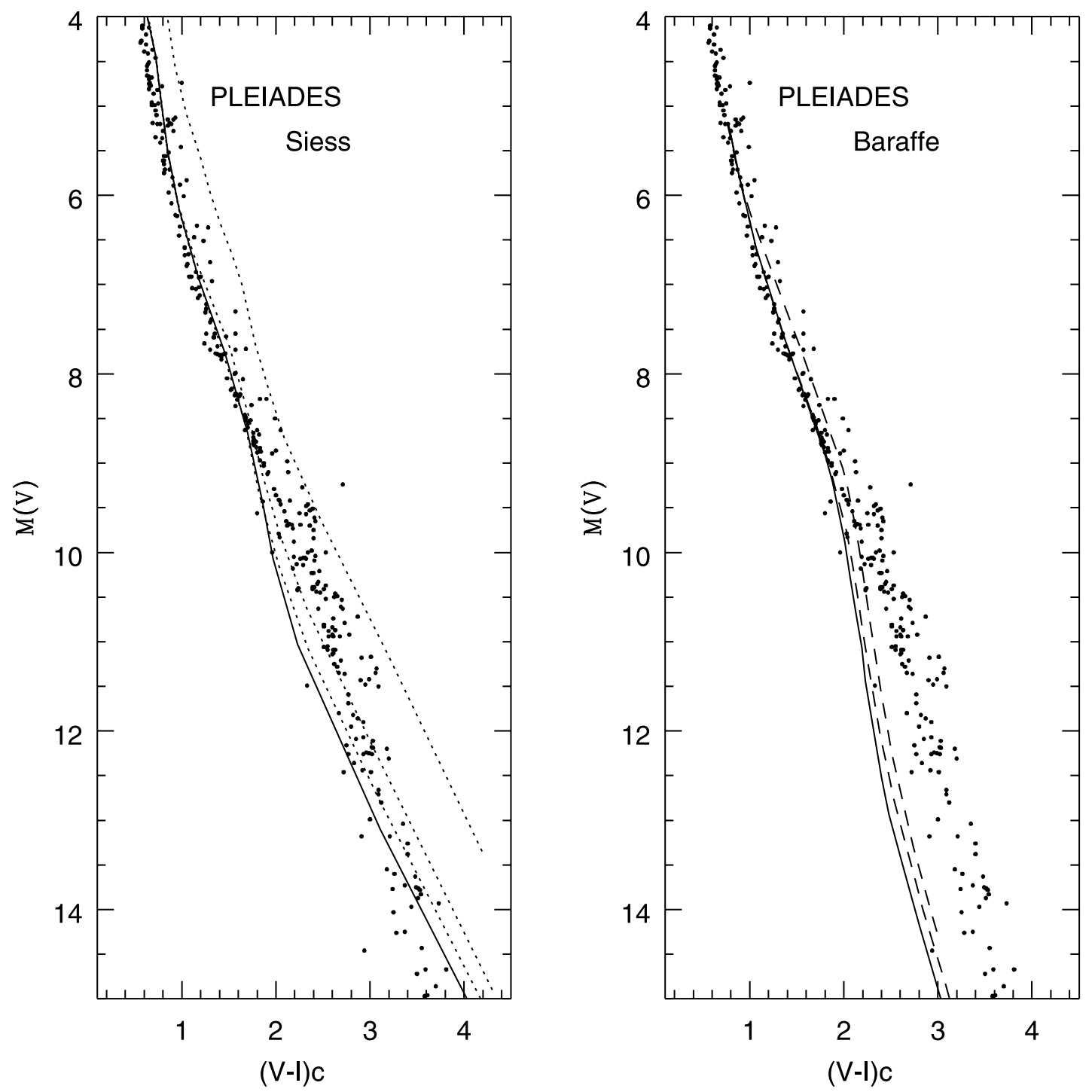

FIG. 13. $-V$ vs. $(V-I)_{c}$ CMD for Pleiades candidate members from Table 2 for which we have photoelectric photometry, compared to theoretical isochrones from

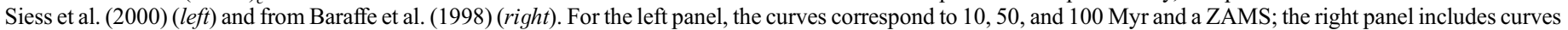
for 50 and 100 Myr and a ZAMS.

appearance of the Pleiades stars in two of these diagrams, and the single-star locus we have defined. The curve defining the singlestar locus was drawn entirely "by eye." It is displaced slightly above the lower envelope to the locus of stars to account for photometric uncertainties (which increase to fainter magnitudes). We attempted to use all of the information available to us, however. That is, there should also be an upper envelope to the Pleiades locus in these diagrams, since equal-mass binaries should be displaced above the single-star sequence by 0.7 mag (and one expects very few systems of higher multiplicity). Therefore, the single-star locus was defined with that upper envelope in mind. Table 4 provides the single-star loci for the Pleiades for $B V I_{\mathrm{C}} J K_{s}$ plus the four IRAC channels. We have dereddened the empirical loci by the canonical mean extinction to the Pleiades of $A_{V}=$ 0.12 (and, correspondingly, $A_{B}=0.16, A_{I}=0.07, A_{J}=0.03$, and $A_{K}=0.01$, as per the reddening law of Rieke \& Lebofsky 1985).

The other benefit to constructing the new catalog is that it can provide an improved comparison data set to test theoretical isochrones. The new catalog provides homogeneous photometry in many photometric bands for stars ranging from several solar masses down to below $0.1 M_{\odot}$. We take the distance to the Pleiades as $133 \mathrm{pc}$ and refer the reader to Soderblom et al. (2005) for a discussion and a listing of the most recent determinations. The age of the Pleiades is not as well-defined but is probably somewhere between 100 and $125 \mathrm{Myr}$ (Meynet et al. 1993; Stauffer et al. 1999). We adopt $100 \mathrm{Myr}$ for the purposes of this discussion; our conclusions relative to the theoretical isochrones would not be affected significantly if we instead chose 125 Myr. As noted above, we adopt $A_{V}=0.12$ as the mean Pleiades extinction and apply that value to the theoretical isochrones. A small number of Pleiades members have significantly larger extinctions (Breger 1986; Stauffer \& Hartmann 1987), and we have dereddened those stars individually to the mean cluster reddening.

Figures 13 and 14 compare theoretical $100 \mathrm{Myr}$ isochrones from Siess et al. (2000) and Baraffe et al. (1998) to the Pleiades member photometry from Table 2 for stars for which we have photoelectric photometry. Neither set of isochrones are a good fit to the $V-I$ based color-magnitude diagram. For Baraffe et al. (1998) this is not a surprise because they illustrated that their isochrones are too blue in $V-I$ for cool stars in their paper and ascribed the problem as likely the result of an incomplete line list, 

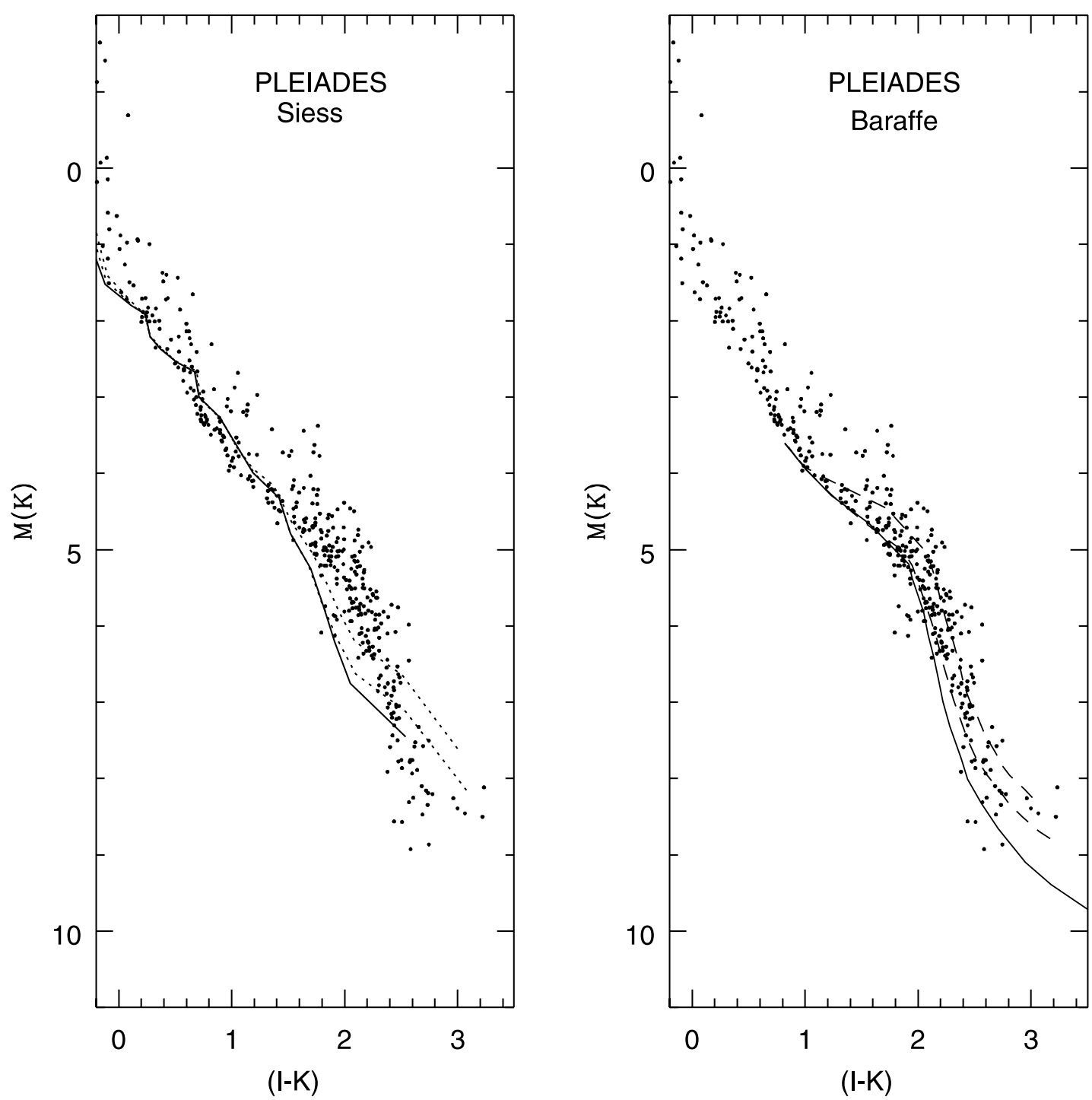

Fig. 14. $-K$ vs. $(I-K)$ CMD for Pleiades candidate members from Table 2, compared to theoretical isochrones from Siess et al. (2000) (left) and from Baraffe et al. (1998) (right). The curves correspond to 50 and $100 \mathrm{Myr}$ and a ZAMS.

resulting in too little absorption in the $V$ band. For Siess et al. (2000) the poor fit in the $V-I \mathrm{CMD}$ is somewhat unexpected in that they transform from the theoretical to the observational plane using empirical color-temperature relations. In any event, it is clear that neither model isochrones match the shape of the Pleiades locus in the $V$ versus $V-I$ plane, and therefore use of these $V-I$ based isochrones for younger clusters is not likely to yield accurate results (unless the color- $T_{\text {eff }}$ relation is recalibrated, as described, e.g., in Jeffries \& Oliveira 2005). On the other hand, the Baraffe et al. (1998) model provides a quite good fit to the Pleiades single-star locus for an age of $100 \mathrm{Myr}$ in the $K$ versus $I-K$ plane. ${ }^{6}$ This perhaps lends support to the hypothesis that the misfit in the $V$ versus $V-I$ plane is due to missing opacity in their $V$-band atmospheres for low-mass stars (see also Chabrier et al. 2000 for further evidence in support of this idea). The Siess et al. (2000) isochrones do not fit the Pleiades locus in the $K$ versus

\footnotetext{
${ }^{6}$ These isochrones are calculated for the standard $K$ filter, rather than $K_{s}$. However, the difference in location of the isochrones in these plots because of this should be very slight, and we do not believe our conclusions are significantly affected.
}

$I-K$ plane particularly well, being too faint near $I-K=2$ and too bright for $I-K>2.5$.

\section{IDENTIFICATION OF NEW VERY LOW-MASS CANDIDATE MEMBERS}

The highest spatial density for Pleiades members of any mass should be at the cluster center. However, searches for substellar members of the Pleiades have generally avoided the cluster center because of the deleterious effects of scattered light from the high-mass cluster members and because of the variable background from the Pleiades reflection nebulae. The deep 2MASS and IRAC $3.6 \mu \mathrm{m}$ imaging and $4.5 \mu \mathrm{m}$ imaging provide accurate photometry to well below the hydrogen-burning mass limit and are less affected by the nebular emission than shorter wavelength images. We therefore expect that it should be possible to identify a new set of candidate Pleiades substellar members by combining our new near- and mid-infrared photometry.

The substellar mass limit in the Pleiades occurs at about $K_{s}=$ 14.4, near the limit of the 2MASS All-Sky PSC. As illustrated in Figure 15, the deep 2MASS survey of the Pleiades should easily detect objects at least 2 mag fainter than the substellar limit. The 


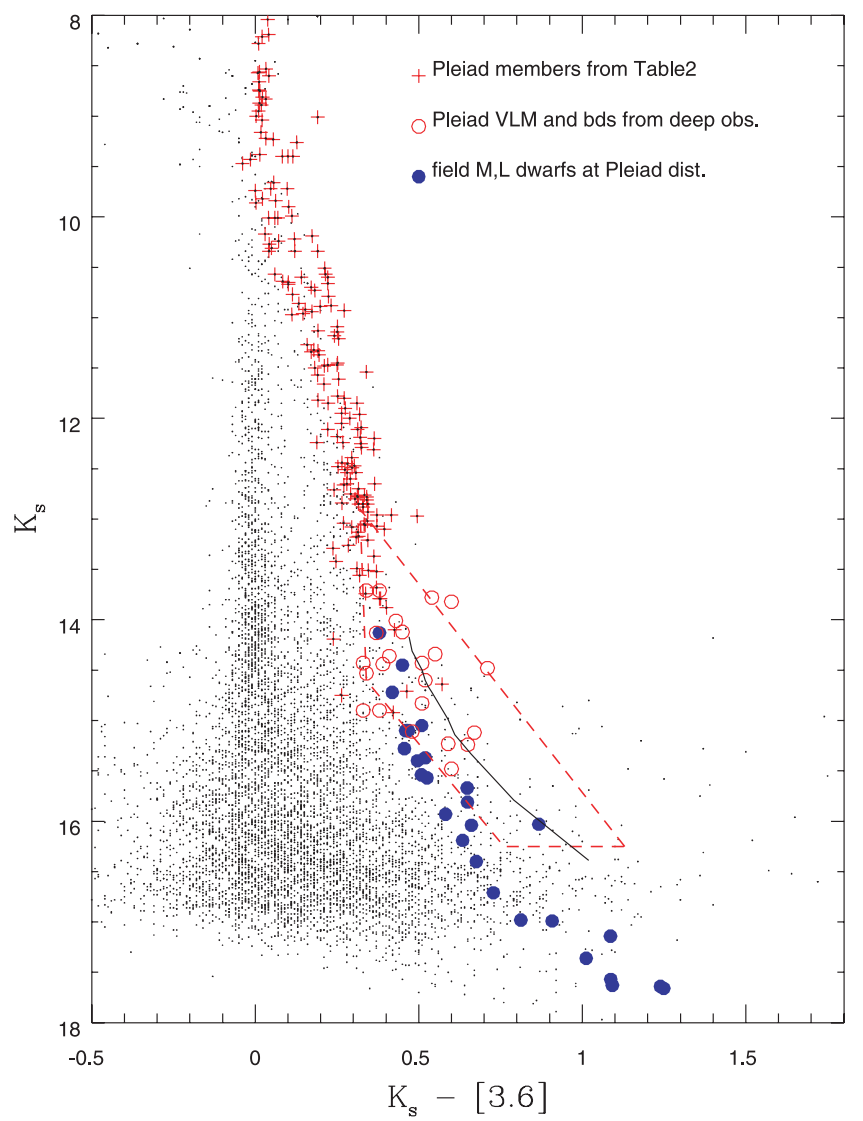

Fig. 15. $-K_{s}$ vs. $K_{s}-[3.6]$ CMD for the objects in the central $1 \mathrm{deg}^{2}$ of the Pleiades, combining data from the IRAC shallow survey and 2MASS. The symbols are defined within the figure (and see text for details). The dashed-line box indicates the region within which we have searched for new candidate Pleiades VLM and substellar members. The solid curve is a DUSTY $100 \mathrm{Myr}$ isochrone from Chabrier et al. (2000) for masses from 0.1 to $0.03 M_{\odot}$

key to actually identifying those objects and separating them from the background sources is to find color-magnitude or colorcolor diagrams that separate the Pleiades members from the other objects. As shown in Figure 15, late-type Pleiades members separate fairly well from most field stars toward the Pleiades in a $K_{s}$ versus $K_{s}-[3.6]$ color-magnitude diagram. However, as illustrated in Figure 2, in the $K_{s}$ magnitude range of interest there is also a large population of red galaxies, and they are in fact the primary contaminants to identifying Pleiades substellar objects in the $K_{s}$ versus $K_{s}-[3.6]$ plane. Fortunately, most of the contaminant galaxies are slightly resolved in the 2MASS and IRAC imaging, and we have found that we can eliminate most of the red galaxies by their nonstellar image shape.

Figure 15 shows the first step in our process of identifying new very low-mass members of the Pleiades. The red plus symbols are the known Pleiades members from Table 2. The red open circles are candidate Pleiades substellar members from deep imaging surveys published in the literature, mostly of parts of the cluster exterior to the central square degree, where the IRAC photometry is from P. Lowrance et al. (2007, in preparation). The blue, filled circles are field $\mathrm{M}$ and $\mathrm{L}$ dwarfs, placed at the distance of the Pleiades, using photometry from Patten et al. (2006). Because the Pleiades is $\sim 100 \mathrm{Myr}$, its very low-mass stellar and substellar objects will be displaced about $0.7 \mathrm{mag}$ above the locus of the field M and L dwarfs according to the Baraffe et al. (1998) and Chabrier et al. (2000) models, in accord with the location in the diagram of the previously identified, candidate VLM and substellar objects. The trapezoidal shaped region outlined with a dashed line is the region in the diagram that we define as containing candidate new VLM and substellar members of the Pleiades. We place the faint limit of this region at $K_{s}=16.2$ in order to avoid the large apparent increase in faint, red objects for $K_{s}>$ 16.2 , caused largely by increasing errors in the $K_{s}$ photometry. Also, the 2MASS extended object flags cease to be useful fainter than about $K_{s}=16$.

We took the following steps to identify a set of candidate substellar members of the Pleiades:

1. keep only objects that fall in the trapezoidal region in Figure 15 ;

2. remove objects flagged as nonstellar by the 2MASS pipeline software;

3. remove objects that appear nonstellar to the eye in the IRAC images;

4. remove objects that do not fall in or near the locus of field $\mathrm{M}$ and $\mathrm{L}$ dwarfs in a $J-H$ versus $H-K_{S}$ diagram;

5. remove objects that have 3.6 and $4.5 \mu$ m magnitudes that differ by more than $0.2 \mathrm{mag}$;

6. remove objects that fall below the ZAMS in a $J$ versus $J-K_{s}$ diagram.

As shown in Figure 15, all stars earlier than about mid-M have $K_{s}-[3.6]$ colors bluer than 0.4. This ensures that for most of the area of the trapezoidal region, the primary contaminants are distant galaxies. Fortunately, the 2MASS catalog provides two types of flags for identifying extended objects. For each filter, a $\chi^{2}$ flag measures the match between the objects shape and the instrumental PSF, with values greater than 2.0 generally indicative of a nonstellar object. In order not to be misguided by an image artifact in one filter, we throw out the most discrepant of the three flags and average the other two. We discard objects with mean $\chi^{2}$ greater than 1.9. The other indicator is the 2MASS extended object flag, which is the synthesis of several independent tests of the objects shape, surface brightness and color (see Jarrett et al. 2000 for a description of this process). If one simply excludes the objects classified as extended in the 2MASS 6x image by either of these techniques, the number of candidate VLM and substellar objects lying inside the trapezoidal region decreases by nearly a half.

We have one additional means to demonstrate that many of the identified objects are probably Pleiades members, and that is via proper motions. The mean Pleiades proper motion is $\Delta$ R.A. $=$ $20 \mathrm{mas} \mathrm{yr}^{-1}$ and $\Delta$ decl. $=-45 \mathrm{mas} \mathrm{yr}^{-1}$ (Jones 1973). With an epoch difference of only $3.5 \mathrm{yr}$ between the deep 2MASS and IRAC imaging, the expected motion for a Pleiades member is only $0.07^{\prime \prime}$ in right ascension and $-0.16^{\prime \prime}$ in declination. Given the relatively large pixel size for the two cameras, and the undersampled nature of the IRAC 3.6 and $4.5 \mu \mathrm{m}$ images, it is not a priori obvious that one would expect to reliably detect the Pleiades motion. However, both the 2MASS and IRAC astrometric solutions have been very accurately calibrated. Also, for the present purpose, we only ask whether the data support a conclusion that most of the identified substellar candidates are true Pleiades members (i.e., as an ensemble), rather than that each star is well enough separated in a VPD to derive a high membership probability.

Figure 16 provides a set of plots that we believe support the conclusion that the majority of the surviving VLM and substellar candidates are Pleiades members. The first plot shows the measured motions between the epoch of the 2MASS and IRAC observations for all known Pleiades members from Table 2 that lie in 

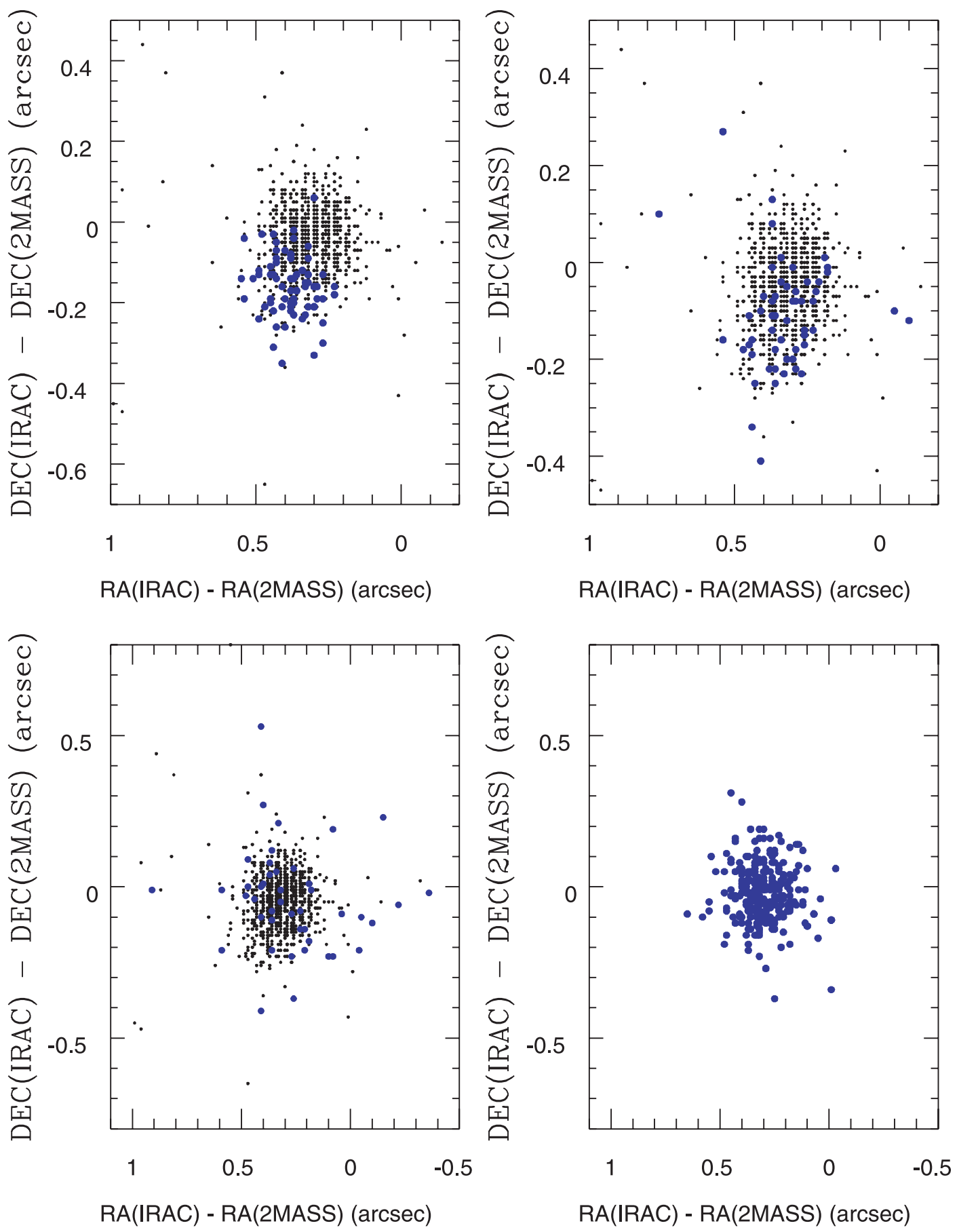

FIG. 16.-Proper-motion vector point diagrams (VPDs) for various stellar samples in the central $1^{\circ}$ field, derived from combining the IRAC and 2MASS 6x observations. Top left: VPD comparing all objects in the field (small black dots) to Pleiades members with $11<K_{s}<14$ (large blue dots). Top right: Same, except the blue dots are the new candidate VLM and substellar Pleiades members. Bottom left: Same, except the blue dots are a nearby, low-mass field star sample from a box just blueward of the trapezoidal region in 15. Bottom right: VPD just showing a second, distant field star sample as described in the text.

the central square degree region and have $11<K_{s}<14$ (i.e., just brighter than the substellar candidates). The mean offset of the Pleiades stellar members from the background population is welldefined and is quantitatively of the expected magnitude and sign $\left(+0.07^{\prime \prime}\right.$ in right ascension and $-0.16^{\prime \prime}$ in declination). The rms dispersion of the coordinate difference for the field population in right ascension and declination is $0.076^{\prime \prime}$ and $0.062^{\prime \prime}$, supportive of our claim that the relative astrometry for the two cameras is quite good. Because we expect that the background population should have essentially no mean proper motion, the nonzero mean "motion" of the field population of about $\langle\Delta$ R.A. $\rangle=0.3$ " is presumably not real. Instead, the offset is probably due to the uncertainty in transferring the Spitzer coordinate zero point between the warm star-tracker and the cryogenic focal plane. Because it is simply a zero-point offset applicable to all the objects in the IRAC catalog, it has no effect on the ability to separate Pleiades members from the field star population.

The second panel in Figure 16 shows the proper motion of the candidate Pleiades VLM and substellar objects. While these objects do not show as clean a distribution as the known members, 


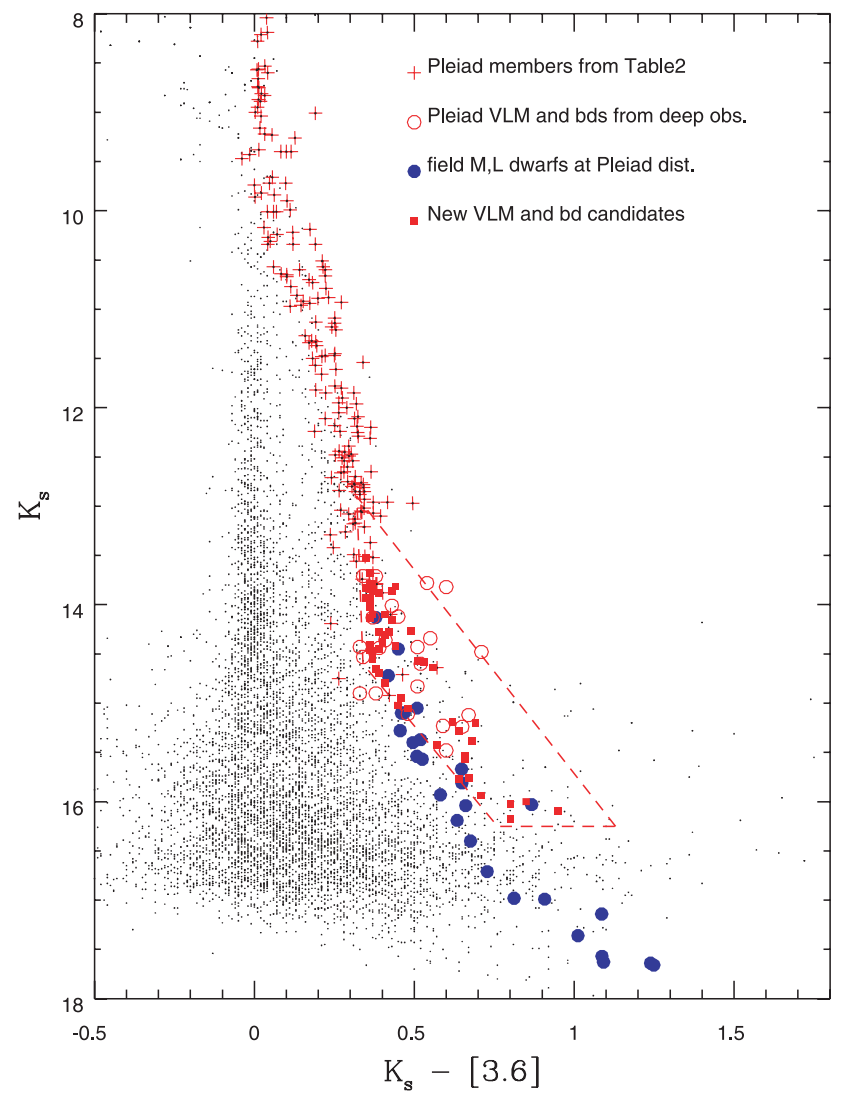

FIG. 17.- Same as Fig. 15, except that the new candidate VLM and substellar objects from Table 5 are now indicated as small, red squares.

their mean motion is clearly in the same direction. After removing $2 \sigma$ deviants, the median offsets for the substellar candidates are $0.04^{\prime \prime}$ and $-0.11^{\prime \prime}$ in right ascension and declination, respectively. The objects whose motions differ significantly from the Pleiades mean may be nonmembers or they may be members with poorly determined motions (since a few of the high-probability members in the first panel also show discrepant motions).

The other two panels in Figure 16 show the proper motions of two possible control samples. The first control sample was defined as the set of stars that fall up to $0.3 \mathrm{mag}$ below the lower sloping boundary of the trapezoid in Figure 15. These objects should be late-type dwarfs that are either older or more distant than the Pleiades or red galaxies. We used the 2MASS data to remove extended or blended objects from the sample in the same way as for the Pleiades candidates. If the objects are nearby field stars, we expect to see large proper motions; if galaxies, the real proper motions would be small - but relatively large apparent proper motions due to poor centroiding or different centroids at different effective wavelengths could be present. The second control set was defined to have $-0.1<K-[3.6]<0.1$ and $14.0<K<$ 14.5 and to be stellar based on the 2MASS flags. This control sample should therefore be relatively distant $\mathrm{G}$ and $\mathrm{K}$ dwarfs primarily. Both control samples have proper-motion distributions that differ greatly from the Pleiades samples and that make sense for, respectively, a nearby and a distant field star sample.

Figure 17 shows the Pleiades members from Table 2 and the 55 candidate VLM and substellar members that survived all of our culling steps. We cross-correlated this list with the stars from Table 2 and with a list of the previously identified candidate substellar members of the cluster from other deep imaging surveys. Fourteen of the surviving objects correspond to previously iden- tified Pleiades VLM and substellar candidates. We provide the new list of candidate members in Table 5. The columns marked as $\mu$ (R.A.) and $\mu$ (decl.) are the measured motions in arcsec over the $3.5 \mathrm{yr}$ epoch difference between the 2MASS-6x and IRAC observations. Forty-two of these objects have $K_{s}>14.0$ and hence inferred masses less than about $0.1 M_{\odot} ; 31$ of them have $K_{s}>14.4$ and hence have inferred masses below the hydrogenburning mass limit.

Our candidate list could be contaminated by foreground latetype dwarfs that happen to lie in the line of sight to the Pleiades. How many such objects should we expect? In order to pass our culling steps, such stars would have to be mid- to late-M dwarfs, or early to mid-L dwarfs. We use the known $\mathrm{M}$ dwarfs within $8 \mathrm{pc}$ to estimate how many field $\mathrm{M}$ dwarfs should lie in a $1 \mathrm{deg}^{2} \mathrm{re}-$ gion and at distance between 70 and $100 \mathrm{pc}$ (so they would be coincident in a CMD with the $100 \mathrm{Myr}$ Pleiades members). The result is $\sim 3$ such field $\mathrm{M}$ dwarf contaminants. Cruz et al. (2007) estimate that the volume density of L dwarfs is comparable to that for late-M dwarfs, and therefore a very conservative estimate is that there might also be 3 field $\mathrm{L}$ dwarfs contaminating our sample. We regard this (6 contaminating field dwarfs) as an upper limit because our various selection criteria would exclude early-M dwarfs and late-L dwarfs. Bihain et al. (2006) made an estimate of the number of contaminating field dwarfs in their Pleiades survey of $1.8 \mathrm{deg}^{2}$; for the spectral type range of our objects, their algorithm would have predicted just one or two contaminating field dwarfs for our survey.

How many substellar Pleiades members should there be in the region we have surveyed? That is, of course, part of the question we are trying to answer. However, previous studies have estimated that the Pleiades stellar mass function for $M<0.5 M_{\odot}$ can be approximated as a power law with an exponent of $-1(d N / d M \propto$ $M^{-1}$ ). Using the known Pleiades members from Table 2 that lie within the region of the IRAC survey and that have masses of $0.2<M / M_{\odot}<0.5$ (as estimated from the Baraffe et al. (1998) $100 \mathrm{Myr}$ isochrone) to normalize the relation, the $M^{-1}$ mass function predicts about 48 members in our search region and with $14<K<16.2$ (corresponding to $0.1<M / M_{\odot}<0.035$ ). Other studies have suggested that the mass function in the Pleiades becomes shallower below $0.1 M_{\odot}, d N / d M \propto M^{-0.6}$. Using the same normalization as above, this functional form for the Pleiades mass function for $M<0.1 M_{\odot}$ yields a prediction of $20 \mathrm{VLM}$ and substellar members in our survey. The number of candidates we have found falls between these two estimates. Better proper motions and low-resolution spectroscopy will almost certainly eliminate some of these candidates as nonmembers.

\section{MID-IR OBSERVATIONS OF DUST AND POLYCYCLIC AROMATIC HYDROCARBONS IN THE PLEIADES}

Since the earliest days of astrophotography, it has been clear that the Pleiades stars are in relatively close proximity to interstellar matter whose optical manifestation is the spider-web-like network offilaments seen particularly strongly toward several of the B stars in the cluster. High-resolution spectra of the brightest Pleiades stars as well as CO maps toward the cluster show that there is gas as well as dust present and that the (primary) interstellar cloud has a significant radial velocity offset relative to the Pleiades (White 2003; Federman \& Willson 1984). The gas and dust, therefore, are not a remnant from the formation of the cluster but are simply evidence of a transitory event as this small cloud passes by the cluster in our line of sight (see also Breger 1986). There are at least two claimed morphological signatures of a direct interaction of the Pleiades with the cloud. White \& Bally (1993) provided evidence that the IRAS 60 and $100 \mu \mathrm{m}$ image of the 
TABLE 5

New Candidate Pleiades Members

\begin{tabular}{|c|c|c|c|c|c|c|c|c|c|c|}
\hline ID & $\begin{array}{l}\text { R.A. (J2000.0) } \\
\text { (deg) }\end{array}$ & $\begin{array}{c}\text { Decl. (J2000.0) } \\
\text { (deg) }\end{array}$ & $J$ & $H$ & $K_{s}$ & {$[3.6]$} & {$[4.5]$} & $\mu$ (R.A.) & $\mu($ decl. $)$ & Previous ID \\
\hline SI2M-1 …........................ & 56.15745 & 24.42746 & 14.44 & 13.79 & 13.52 & 13.17 & 13.10 & 0.37 & -0.01 & HHJ 46 \\
\hline SI2M-2 ...................... & 56.19235 & 24.38414 & 14.68 & 14.10 & 13.79 & 13.42 & 13.36 & 0.44 & -0.19 & HHJ 24 \\
\hline SI2M-3 ....................... & 56.24477 & 24.27201 & 17.85 & 16.83 & 16.00 & 15.15 & 15.15 & 0.37 & 0.13 & $\ldots$ \\
\hline SI2M-4 ........................ & 56.28952 & 23.97910 & 15.46 & 14.83 & 14.41 & 14.05 & 14.05 & 0.54 & -0.16 & $\ldots$ \\
\hline SI2M-5 ......................... & 56.29098 & 24.07576 & 14.80 & 14.16 & 13.86 & 13.43 & 13.37 & 0.45 & -0.17 & \\
\hline SI2M-6 ....................... & 56.30265 & 23.89584 & 14.83 & 14.21 & 13.88 & 13.49 & 13.47 & 0.37 & -0.14 & HHJ 14 \\
\hline SI2M-7 ........................ & 56.32663 & 23.87112 & 15.96 & 15.15 & 14.79 & 14.38 & 14.34 & 0.18 & -0.01 & $\ldots$ \\
\hline SI2M-8 ......................... & 56.36751 & 24.52373 & 16.84 & 16.05 & 15.44 & $\ldots$ & 14.79 & 0.32 & -0.05 & $\ldots$ \\
\hline SI2M-9 ....................... & 56.39588 & 23.85472 & 15.78 & 15.02 & 14.65 & 14.27 & 14.18 & 0.37 & 0.08 & $\ldots$ \\
\hline SI2M-10 ........................ & 56.40739 & 23.73057 & 14.79 & 14.15 & 13.81 & 13.37 & 13.40 & 0.33 & -0.23 & $\ldots$ \\
\hline SI2M-11 ...................... & 56.42205 & 23.90273 & 15.39 & 14.73 & 14.28 & 13.86 & 13.85 & 0.41 & -0.10 & $\ldots$ \\
\hline SI2M-12 ..................... & 56.42644 & 24.06976 & 15.27 & 14.64 & 14.28 & 13.89 & 13.95 & 0.36 & -0.18 & $\ldots$ \\
\hline SI2M-13 ..................... & 56.43118 & 23.64760 & 15.17 & 14.43 & 14.14 & 13.78 & 13.76 & 0.36 & -0.22 & $\ldots$ \\
\hline SI2M-14 .......................... & 56.44669 & 24.51118 & 17.25 & 16.37 & 15.75 & & 15.04 & 0.43 & -0.25 & $\ldots$ \\
\hline SI2M-15 ....................... & 56.45366 & 23.64644 & 17.53 & 16.49 & 15.57 & 14.91 & 14.62 & 0.21 & -0.04 & $\ldots$ \\
\hline SI2M-16 ..................... & 56.45598 & 23.95163 & 14.70 & 14.07 & 13.83 & 13.48 & 13.36 & 0.37 & -0.08 & $\ldots$ \\
\hline SI2M-17 ...................... & 56.45634 & 24.26979 & 18.11 & 16.71 & 16.18 & 15.38 & 15.21 & 0.76 & 0.10 & $\ldots$ \\
\hline SI2M-18 ………............ & 56.46099 & 23.74362 & 16.39 & 15.70 & 15.28 & 14.64 & 14.79 & 0.34 & 0.01 & $\ldots$ \\
\hline SI2M-19 ……......... & 56.46113 & 24.15099 & 15.81 & 15.06 & 14.64 & 14.08 & 14.02 & 0.30 & -0.20 & BPL 79 \\
\hline SI2M-20 ..................... & 56.46912 & 23.86272 & 15.32 & 14.64 & 14.31 & 13.90 & 13.78 & 0.47 & -0.18 & $\ldots$ \\
\hline SI2M-21 ....................... & 56.47910 & 23.56604 & 15.57 & 14.96 & 14.55 & 14.18 & $\ldots$ & 0.44 & -0.34 & \\
\hline SI2M-22 ..................... & 56.49051 & 24.05142 & 14.72 & 14.12 & 13.79 & 13.43 & 13.44 & 0.37 & -0.11 & HHJ 27 \\
\hline SI2M-23 ..................... & 56.49128 & 24.41130 & 16.74 & 16.09 & 15.54 & 14.88 & 14.82 & 1.17 & 0.10 & $\ldots$ \\
\hline SI2M-24 ...................... & 56.49132 & 24.14474 & 14.58 & 13.99 & 13.68 & 13.32 & 13.32 & 0.22 & -0.06 & DH 412 \\
\hline SI2M-25 ...................... & 56.52133 & 23.75971 & 15.56 & 14.81 & 14.38 & 13.98 & 13.93 & 0.29 & -0.08 & $\ldots$ \\
\hline SI2M-26 ...................... & 56.52526 & 23.97200 & 14.88 & 14.22 & 13.93 & 13.58 & 13.53 & 0.26 & -0.14 & $\ldots$ \\
\hline SI2M-27 .................... & 56.57735 & 23.98407 & 14.75 & 14.11 & 13.86 & 13.49 & 13.39 & 0.30 & -0.08 & $\ldots$ \\
\hline SI2M-28 ..................... & 56.57843 & 23.81347 & 15.83 & 15.02 & 14.57 & 14.06 & 14.18 & 0.29 & -0.18 & $\ldots$ \\
\hline SI2M-29 ....................... & 56.58151 & 23.56235 & 15.80 & 15.08 & 14.69 & 14.30 & $\ldots$ & 0.23 & -0.14 & $\ldots$ \\
\hline SI2M-30 ......................... & 56.58557 & 24.28870 & 17.05 & 16.31 & 15.76 & 15.09 & 14.87 & -0.10 & -0.12 & $\ldots$ \\
\hline SI2M-31 ....................... & 56.59283 & 23.87408 & 15.59 & 14.94 & 14.46 & 14.10 & 14.01 & 0.45 & -0.11 & \\
\hline SI2M-32 …..................... & 56.60060 & 24.50354 & 14.75 & 14.14 & 13.84 & 13.48 & 13.42 & 0.38 & -0.22 & BPL 101 \\
\hline SI2M-33 ..................... & 56.60880 & 24.08598 & 15.19 & 14.52 & 14.15 & 13.72 & 13.74 & 0.40 & -0.07 & $\ldots$ \\
\hline SI2M-34 ..................... & 56.63392 & 24.38740 & 17.25 & 16.34 & 15.77 & 15.13 & 15.11 & 0.27 & -0.23 & $\ldots$ \\
\hline SI2M-35 ..................... & 56.64737 & 23.95206 & 15.37 & 14.77 & 14.45 & 14.06 & 13.97 & 0.29 & -0.22 & $\ldots$ \\
\hline SI2M-36 ...................... & 56.67914 & 24.41405 & 15.55 & 14.85 & 14.42 & 13.98 & 14.00 & 0.26 & -0.17 & BPL 108 \\
\hline SI2M-37 .................... & 56.70850 & 24.00659 & 15.69 & 14.98 & 14.58 & 14.05 & 14.00 & 0.34 & -0.04 & $\ldots$ \\
\hline 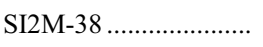 & 56.75776 & 24.22451 & 14.97 & 14.41 & 14.10 & 13.73 & 13.62 & 0.29 & -0.06 & BPL 122 \\
\hline SI2M-39 ..................... & 56.77373 & 24.66767 & 15.47 & 14.87 & 14.51 & $\ldots$ & 14.01 & 0.36 & -0.07 & $\ldots$ \\
\hline SI2M-40 ..................... & 56.79400 & 23.90606 & 15.99 & 15.28 & 15.02 & 14.57 & 14.54 & 0.19 & 0.01 & $\ldots$ \\
\hline SI2M-41 ..................... & 56.79446 & 23.97119 & 14.95 & 14.38 & 14.02 & 13.66 & 13.66 & 0.32 & -0.20 & $\ldots$ \\
\hline SI2M-42 .................... & 56.79918 & 24.22539 & 14.86 & 14.23 & 13.88 & 13.49 & 13.38 & 0.27 & -0.08 & BPL 130 \\
\hline SI2M-43 ..................... & 56.80051 & 24.47547 & 16.19 & 15.53 & 15.05 & 14.57 & 14.65 & 0.41 & -0.41 & BPL 132 \\
\hline SI2M-44...................... & 56.82203 & 24.20922 & 17.54 & 17.00 & 15.94 & 15.23 & 14.62 & -0.05 & -0.10 & $\ldots$ \\
\hline SI2M-45 ..................... & 56.96009 & 23.91330 & 16.41 & 15.71 & 15.20 & 14.51 & 14.53 & 0.34 & -0.16 & $\ldots$ \\
\hline SI2M-46 .................... & 56.96365 & 23.73669 & 17.52 & 16.67 & 16.02 & 15.22 & 15.11 & 0.25 & -0.04 & $\ldots$ \\
\hline SI2M-47..................... & 57.00899 & 24.42107 & 16.75 & 16.05 & 15.42 & 14.85 & 14.76 & 0.23 & -0.08 & $\ldots$ \\
\hline SI2M-48 ..................... & 57.01952 & 23.65838 & 15.28 & 14.66 & 14.27 & 13.78 & $\ldots$ & 0.26 & -0.15 & \\
\hline SI2M-49 .................... & 57.07928 & 24.42024 & 16.02 & 15.27 & 14.95 & 14.49 & 14.51 & 0.36 & -0.11 & BPL 172 \\
\hline SI2M-50 .......................... & 57.09851 & 24.37646 & 14.92 & 14.34 & 13.94 & 13.58 & 13.55 & 0.30 & -0.01 & BPL 177 \\
\hline SI2M-51 ...................... & 57.12811 & 23.70665 & 16.66 & 15.85 & 15.19 & 14.57 & $\ldots$ & 0.32 & -0.12 & $\ldots$ \\
\hline SI2M-52 ..................... & 57.13138 & 24.57707 & 16.78 & 15.88 & 15.38 & 14.70 & 14.65 & 0.18 & -0.02 & $\ldots$ \\
\hline SI2M-53 ........................ & 57.14174 & 24.08293 & 15.38 & 14.67 & 14.47 & 14.10 & 14.00 & 0.44 & -0.16 & $\ldots$ \\
\hline SI2M-54 ..................... & 57.23196 & 24.36115 & 15.04 & 14.43 & 14.10 & 13.69 & 13.66 & 0.36 & -0.25 & HHJ 8 \\
\hline SI2M-55 ......................... & 57.28922 & 23.94612 & 17.70 & 16.77 & 16.09 & 15.14 & 15.02 & 0.54 & 0.27 & $\ldots$ \\
\hline
\end{tabular}

vicinity of the Pleiades showed a dark channel immediately to the east of the Pleiades, which they interpreted as the "wake" of the Pleiades as it plowed through the cloud from the east. Herbig \& Simon (2001) provided a detailed analysis of the optically brightest nebular feature in the Pleiades-IC 349 (Barnard's Merope nebula) - and concluded that the shape and structure of that nebula could best be understood if the cloud was running into the Pleiades from the southeast. Herbig \& Simon (2001) concluded that the IC 349 cloudlet, and by extension the rest of the gas and dust enveloping the Pleiades, are relatively distant outliers of the Taurus molecular clouds (see also Eggen 1950 for a much earlier discussion ascribing the Merope nebulae as outliers 

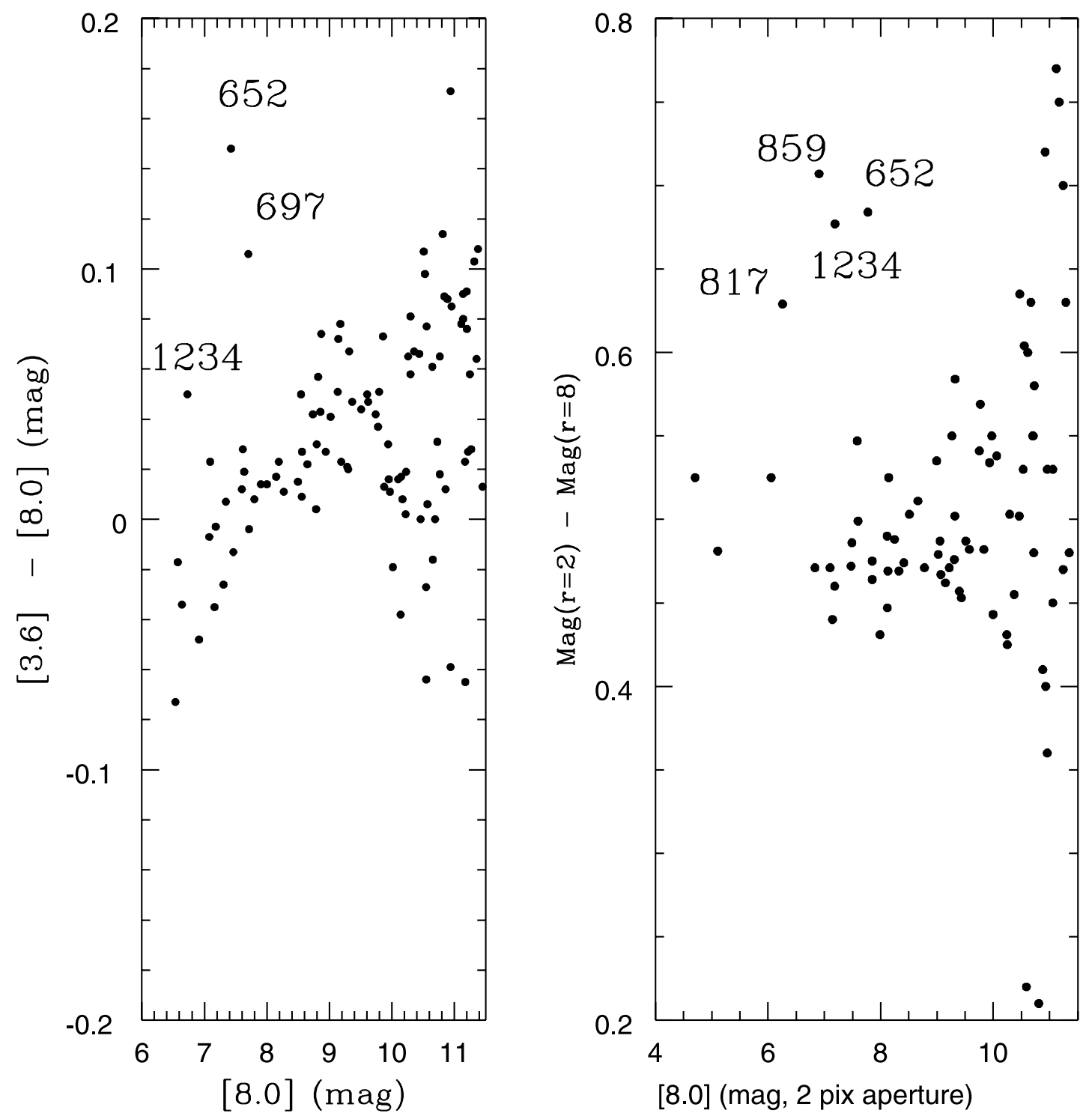

FIG. 18.-Two plots intended to isolate Pleiades members with excess and/or extended $8 \mu$ m emission. The plot with [3.6] $-[8.0] \mu$ m colors shows data from Table 3 (and hence is for aperture sizes of 3 pixel and 2 pixel radius, respectively). The increased vertical spread in the plots at faint magnitudes is simply due to decreasing signalto-noise at $8 \mu \mathrm{m}$. The numbers labeling stars with excesses are the HII identification numbers for those stars.

of the Taurus clouds). White (2003) has more recently proposed a hybrid model, where there are two separate interstellar cloud complexes with very different space motions, both of which are colliding simultaneously with the Pleiades and with each other.

Breger (1986) provided polarization measurements for a sample of member and background stars toward the Pleiades and argued that the variation in polarization signatures across the face of the cluster was evidence that some of the gas and dust was within the cluster. In particular, Figure 6 of that paper showed a fairly distinct interface region, with little residual polarization to the NE portion of the cluster and an L-shaped boundary running EW along the southern edge of the cluster and then north-south along the western edge of the cluster. Stars to the south and west of that boundary show relatively large polarizations and consistent angles (see also our Fig. 5, where we provide a few polarization vectors from Breger 1986 to illustrate the location of the interface region and the fact that the position angle of the polarization correlates well with the location in the interface).

There is a general correspondence between the polarization map and what is seen with IRAC, in the sense that the B stars in the NE portion of the cluster (Atlas and Alcyone) have little nebular emission in their vicinity, whereas those in the western part of the cluster (Maia, Electra, and Asterope) have prominent, filamentary dust emission in their vicinity. The L-shaped boundary is in fact visible in Figure 4 as enhanced nebular emission running between and below a line roughly joining Merope and Electra and then making a right angle and running roughly parallel to a line running from Electra to Maia to HII 1234 (see Fig. 5).

\subsection{Pleiades Dust-Star Encounters Imaged with IRAC}

The Pleiades dust filaments are most strongly evident in IRAC's $8 \mu \mathrm{m}$ channel, as evidenced by the distinct red color of the nebular features in Figure 4. The dominance at $8 \mu \mathrm{m}$ is an expected feature of reflection nebulae, as exemplified by NGC 7023 (Werner et al. 2004), where most of the mid-infrared emission arises from polycyclic aromatic hydrocarbons (PAHs) whose strongest bands in the $3-10 \mu \mathrm{m}$ region fall at 7.7 and $8.6 \mu \mathrm{m}$. One might expect that if portions of the passing cloud were particularly near to one of the Pleiades members, it might be possible to identify such interactions by searching for stars with $8.0 \mu \mathrm{m}$ excesses or for stars with 

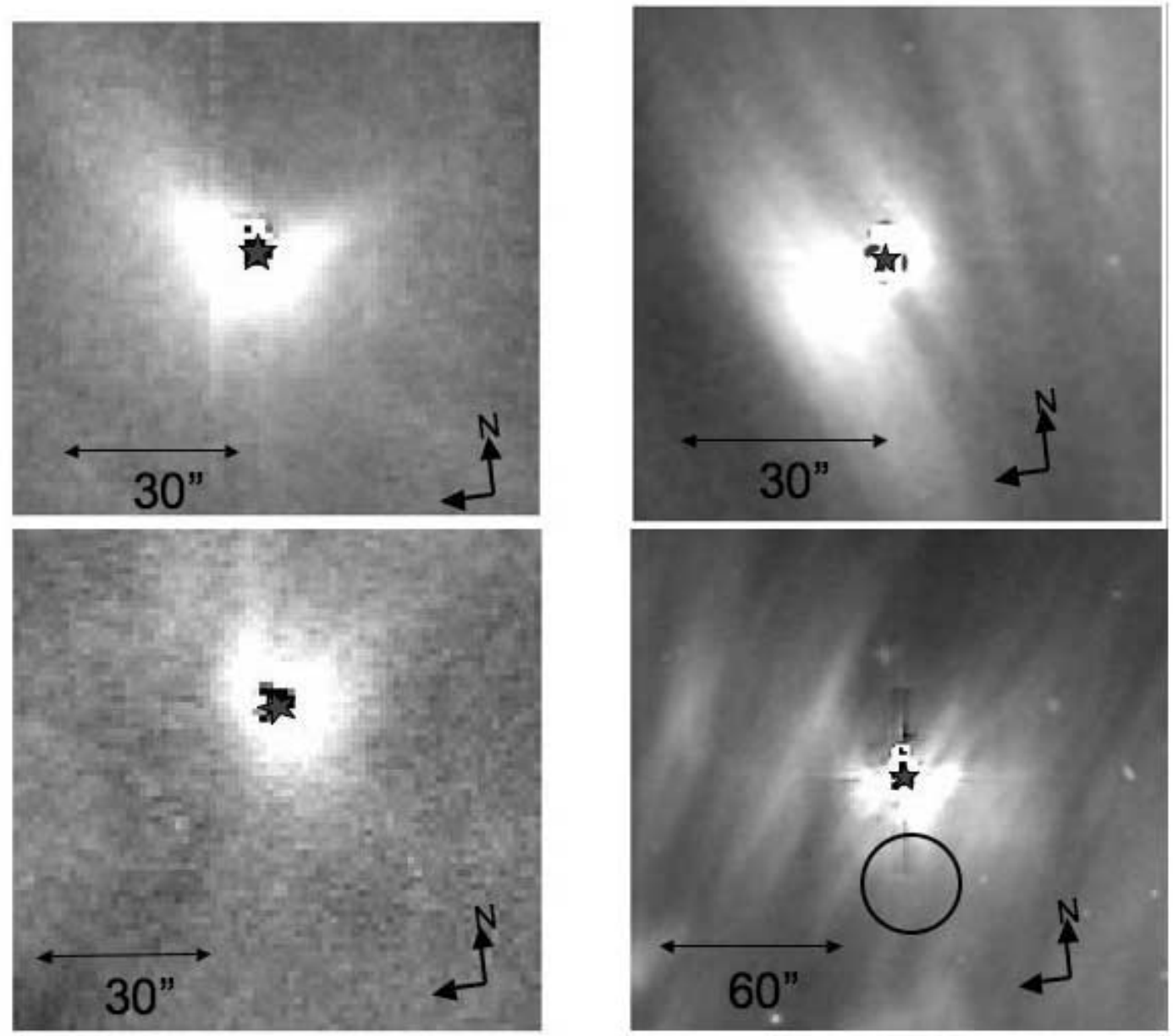

FIG. 19.-Postage stamp images extracted from individual, $8 \mu \mathrm{m}$ BCDs for the stars with extended $8 \mu \mathrm{m}$ emission, from which we have subtracted an empirical PSF. Clockwise from the upper left, the stars shown are HII 1234, HII 859, Merope, and HII 652. The five-pointed star indicates the astrometric position of the star (often superposed on a few black pixels where the $8 \mu \mathrm{m}$ image was saturated. The circle in the Merope image is centered on the location of IC 349 and has a diameter of about $25^{\prime \prime}$ (the size of IC 349 in the optical is of order $10^{\prime \prime} \times 10^{\prime \prime}$ ).

extended emission at $8 \mu \mathrm{m}$. Figure 18 provides two such plots. Four stars stand out as having significant extended $8 \mu \mathrm{m}$ emission, with two of those stars also having an $8 \mu \mathrm{m}$ excess based on their [3.6] - [8.0] color. All of these stars, plus IC 349, are located approximately along the interface region identified by Breger (1986).

We have subtracted a PSF from the $8 \mu \mathrm{m}$ images for the stars with extended emission, and those PSF-subtracted images are provided in Figure 19. The image for HII 1234 has the appearance of a bow shock. The shape is reminiscent of predictions for what one should expect from a collision between a large cloud or a sheet of gas and an A star as described in Artymowicz \& Clampin (1997). The Artymowicz \& Clampin model posits that A stars encountering a cloud will carve a paraboloidal shaped cavity in the cloud via radiation pressure. The exact size and shape of the cavity depend on the relative velocity of the encounter, the star's mass and luminosity and properties of the ISM grains. For typical parameters, the predicted characteristic size of the cavity is of order $1000 \mathrm{AU}$, quite comparable to the size of the structures around HII 652 and HII 1234. The observed appearance of the cavity depends on the view angle to the observer. However, in any case, the direction from which the gas is moving relative to the star can be inferred from the location of the star relative to the curved rim of the cavity; the "wind" originates approximately from the direction connecting the star and the apex of the rim. For HII 1234, this indicates the cloud that it is encountering has a motion relative to HII 1234 from the SSE, in accord with a Taurus origin and not in accord for where a cloud is impacting the Pleiades from the west as posited in White (2003). The nebular emission for HII 652 is less strongly bow-shaped, but the peak of the excess emission is displaced roughly southward from the star, consistent with the Taurus model and inconsistent with gas flowing from the west.

Despite being the brightest part of the Pleiades nebulae in the optical, IC 349 appears to be undetected in the $8 \mu \mathrm{m}$ image. This is not because the $8 \mu \mathrm{m}$ image is insensitive to the nebular emissionthere is generally good agreement between the structures seen in the optical and at $8 \mu \mathrm{m}$, and most of the filaments present in optical images of the Pleiades are also visible on the $8 \mu \mathrm{m}$ image (see Figs. 4 and 19) and even the PSF-subtracted image of Merope shows well-defined nebular filaments. The lack of enhanced $8 \mu \mathrm{m}$ emission from the region of IC 349 is probably because all of the small particles have been scoured away from this cloudlet, consistent with Herbig's model to explain the HST surface photometry and colors. There is no PAH emission from IC 349 because there are none of the small molecules that are the postulated source of the PAH emission.

IC 349 is very bright in the optical, and undetected to a good sensitivity limit at $8 \mu \mathrm{m}$; it must be detectable via imaging at some wavelength between $5000 \AA$ and $8 \mu \mathrm{m}$. We checked our $3.6 \mu \mathrm{m}$ data for this purpose. In the standard BCD mosaic image, 


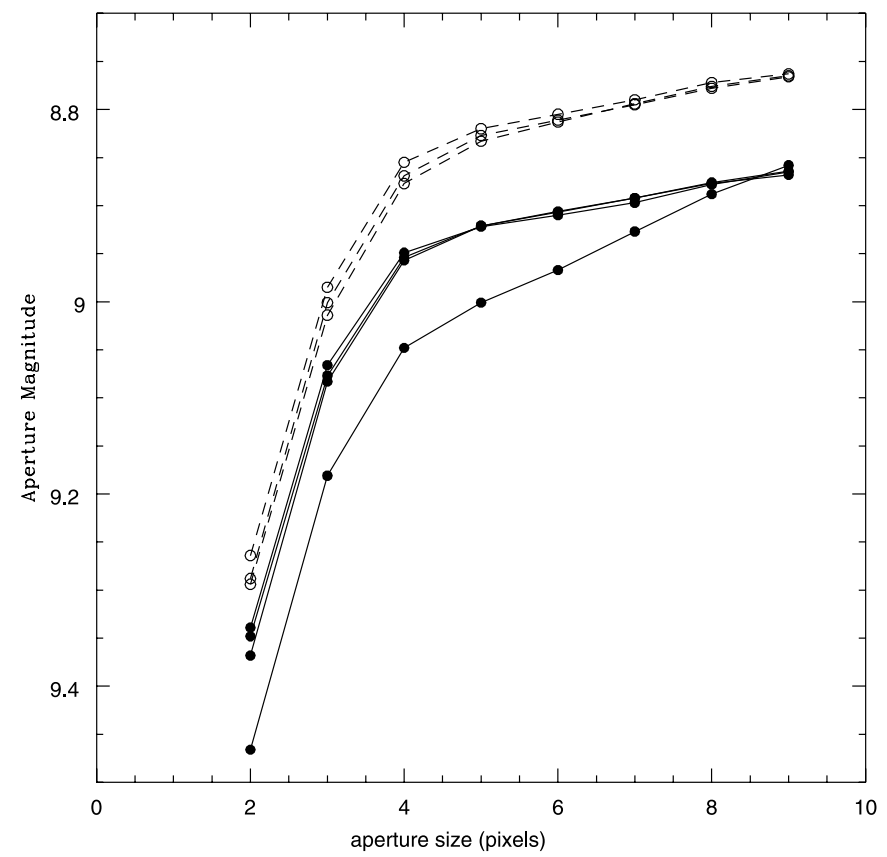

FIG. 20.-Aperture growth curves from the $8 \mu \mathrm{m}$ mosaic for stars with $24 \mu \mathrm{m}$ excesses from Gorlova et al. (2006) and for a set of control objects (dashed curves). All of the objects have been scaled to common zero-point magnitudes for 9 pixel apertures, with the $24 \mu \mathrm{m}$ excess stars offset from the control objects by $0.1 \mathrm{mag}$. The three Gorlova et al. (2006) stars with no excess at $8 \mu \mathrm{m}$ are HII 996, HII 1284, and HII 2195. The Gorlova et al. (2006) star with a slight excess at $8 \mu \mathrm{m}$ is HII 489.

we were unable to discern an excess at the location of IC 349 either simply by displaying the image with various stretches or by doing cuts through the image. We performed a PSF subtraction of Merope from the image in order to attempt to improve our ability to detect faint, extended emission 30" from Meropeunfortunately, bright stars have ghost images in IRAC channel 1, and in this case the ghost image falls almost exactly at the location of IC 349. IC 349 is also not detected in visual inspection of our 2MASS $6 x$ images.

\subsection{Circumstellar Disks and IRAC}

As part of the Spitzer FEPS (Formation and Evolution of Planetary Systems) Legacy program, using pointed MIPS photometry, Stauffer et al. (2005) identified three G dwarfs in the Pleiades as having $24 \mu \mathrm{m}$ excesses probably indicative of circumstellar dust disks. Gorlova et al. (2006) reported results of a MIPS GTO survey of the Pleiades and identified nine cluster members that appear to have $24 \mu \mathrm{m}$ excesses due to circumstellar disks. However, it is possible that in a few cases these apparent excesses could be due instead to a knot of the passing interstellar dust impacting the cluster member or that the $24 \mu \mathrm{m}$ excess could be flux from a background galaxy projected onto the line of sight to the Pleiades member. Careful analysis of the IRAC images of these cluster members may help confirm that the MIPS excesses are evidence for debris disks rather than the other possible explanations.

Six of the Pleiades members with probable $24 \mu \mathrm{m}$ excesses are included in the region mapped with IRAC. However, only four of them have data at $8 \mu \mathrm{m}$ - the other two fall near the edge of the mapped region and only have data at 3.6 and $5.8 \mu \mathrm{m}$. None of the six stars appear to have significant local nebular dust from visual inspection of the IRAC mosaic images. Also, none of them appear problematic in Figure 18. For a slightly more quantitative analysis of possible nebular contamination, we also constructed aperture growth curves for the six stars and compared them to other Pleiades members. All but one of the six show aperture growth curves that are normal and consistent with the expected IRAC PSF. The one exception is HII 489, which has a slight excess at large aperture sizes, as is illustrated in Figure 20. Because HII 489 only has a small $24 \mu \mathrm{m}$ excess, it is possible that the $24 \mu \mathrm{m}$ excess is due to a local knot of the interstellar cloud material and is not due to a debris disk. For the other five $24 \mu \mathrm{m}$ excess stars we find no such problem, and we conclude that their $24 \mu \mathrm{m}$ excesses are indeed best explained as due to debris disks.

\section{SUMMARY AND CONCLUSIONS}

We have collated the primary membership catalogs for the Pleiades to produce the first catalog of the cluster extending from its highest mass members to the substellar limit. At the bright end, we expect this catalog to be essentially complete and with few or no nonmember contaminants. At the faint end, the data establishing membership are much sparser, and we expect a significant number of objects will be nonmembers. We hope that the creation of this catalog will spur efforts to obtain accurate radial velocities and proper motions for the faint candidate members in order to eventually provide a well-vetted membership catalog for the stellar members of the Pleiades. Toward that end, it would be useful to update the current catalog with other data - such as radial velocities, lithium equivalent widths, $\mathrm{X}$-ray fluxes, $\mathrm{H} \alpha$ equivalent widths, etc. - which could be used to help accurately establish membership for the low-mass cluster candidates. It is also possible to make more use of "negative information" present in the propermotion catalogs. That is, if a member from one catalog is not included in another study but does fall within its areal and luminosity coverage, that suggests that it likely failed the membership criteria of the second study. For a few individual stars, we have done this type of comparison, but a systematic analysis of the proper-motion catalogs should be conducted. We intend to undertake these tasks and plan to establish a Web site where these data would be hosted.

We have used the new Pleiades member catalog to define the single-star locus at $100 \mathrm{Myr}$ for $B V I_{\mathrm{C}} K_{s}$ and the four IRAC bands. These curves can be used as empirical calibration curves when attempting to identify members of less well-studied, more distant clusters of similar age. We compared the Pleiades photometry to theoretical isochrones from Siess et al. (2000) and Baraffe et al. (1998). The Siess et al. (2000) isochrones are not, in detail, a good fit to the Pleiades photometry, particularly for low-mass stars. The Baraffe et al. (1998) 100 Myr isochrone does fit the Pleiades photometry very well in the $I$ versus $I-K$ plane.

We have identified 31 new substellar candidate members of the Pleiades using our combined seven-band infrared photometry and have shown that the majority of these objects appear to share the Pleiades proper motion. We believe that most of the objects that may be contaminating our list of candidate brown dwarfs are likely to be unresolved galaxies, and therefore low-resolution spectroscopy should be able to provide a good criterion for culling our list of nonmembers.

The IRAC images, particularly the $8 \mu \mathrm{m}$ mosaic, provide vivid evidence of the strong interaction of the Pleiades stars and the interstellar cloud that is passing through the Pleiades. Our data are supportive of the model proposed by Herbig \& Simon (2001) whereby the passing cloud is part of the Taurus cloud complex and hence is encountering the Pleiades from the SSE direction. White \& Bally (1993) had proposed a model whereby the cloud was encountering the Pleiades from the west and used this to explain 
features in the IRAS 60 and $100 \mu \mathrm{m}$ images of the region as the wake of the Pleiades moving through the cloud. Our data appear to not be supportive of that hypothesis and therefore leave the apparent structure in the IRAS maps as unexplained.

Most of the support for this work was provided by the Jet Propulsion Laboratory, California Institute of Technology, under NASA contract 1407. This research has made use of NASA's Astrophysics Data System (ADS) Abstract Service, and of the SIMBAD database, operated at CDS, Strasbourg, France. This research has made use of data products from the Two Micron All-Sky Survey (2MASS), which is a joint project of the University of Massachusetts and the Infrared Processing and Analysis
Center, funded by the National Aeronautics and Space Administration and the National Science Foundation. These data were served by the NASA/IPAC Infrared Science Archive, which is operated by the Jet Propulsion Laboratory, California Institute of Technology, under contract with the National Aeronautics and Space Administration. The research described in this paper was partially carried out at the Jet Propulsion Laboratory, California Institute of Technology, under contract with the National Aeronautics and Space Administration.

This research made use of the SIMBAD database operated at CDS, Strasbourg, France, and also of the NED and NStED databases operated at IPAC, Pasadena, CA. A large amount of data for the Pleiades (and other open clusters) can also be found at the open cluster database WEBDA (http://www.univie.ac.at/webda/), operated in Vienna by Ernst Paunzen.

\section{APPENDIX}

\section{A1. MEMBERSHIP CATALOGS}

Membership lists of the Pleiades date back to antiquity if one includes historical and literary references to the Seven Sisters (Alcyone, Maia, Merope, Electra, Taygeta, Asterope, and Celeno) and their parents (Atlas and Pleione). The first paper discussing relative proper motions of a large sample of stars in the Pleiades (based on visual observations) was published by Pritchard (1884). The best of the early proper-motion surveys of the Pleiades derived from photographic plate astrometry was that by Trumpler (1921), based on plates obtained at Yerkes and Lick observatories. The candidate members from that survey were presented in two tables, with the first being devoted to candidate members within about $1^{\circ}$ from the cluster center (operationally, within $1^{\circ}$ from Alcyone) and the second table being devoted to candidates further than $1^{\circ}$ from the cluster center. Most of the latter stars were denoted by Trumpler by an S or R, followed by an identification number. We use Tr to designate the Trumpler stars (hence Trnnn for a star from the first table and the small number of stars in the second table without an "S" or an "R," and TrSnnn or TrRnnn for the other stars). For the central region, Trumpler's catalog extends to $V \sim 13$, while the outer region catalog includes stars only to about $V \sim 9$.

The most heavily referenced proper-motion catalog of the Pleiades is that provided by Hertzsprung (1947). That paper makes reference to two separate catalogs: a photometric catalog of the Pleiades published by Hertzsprung (1923), whose members are commonly referred to by HI numbers, and the new proper-motion catalog from the 1947 paper, commonly referenced as the HII catalog. While both HI and HII numbers have been used in subsequent observational papers, it is the HII identification numbers that predominate. That catalog-derived from Carte du Ciel blue-sensitive plates from 14 observatories-includes stars in the central $2^{\circ} \times 2^{\circ}$ region of the cluster and has a faint limit of about $V=15.5$. Johnson system $B V I$ photometry is provided for most of the proposed Hertzsprung members in Johnson \& Mitchell (1958) and Iriarte (1967). Additional Johnson $B$ and $V$ photometry plus Kron $I$ photometry for a fairly large number of the Hertzsprung members can be found in Stauffer (1980, 1982, 1984). Other Johnson $B V$ photometry for a scattering of stars can be found in Jones (1973), Robinson \& Kraft (1974), and Messina (2001). Spectroscopic confirmation, primarily via radial velocities, that these are indeed Pleiades members has been provided in Soderblom et al. (1993), Queloz et al. (1998), and Mermilliod et al. (1997).

Two other proper-motion surveys provide relatively bright candidate members relatively far from the cluster center: Artyukhina \& Kalinina (1970) and van Leeuwen 1986. Stars from the Artyukhina catalog are designated as "AK" followed by the region from which the star was identified followed by an identification number. The new members provided in the van Leeuwen paper were taken from an otherwise unpublished proper-motion study by Pels, where the first 118 stars were considered probable members and the remaining 75 stars were considered possible members. Van Leeuwen categorized a number of the Pels stars as nonmembers based on the Walraven photometry they obtained, and we adopt those findings. Radial velocities for stars in these two catalogs have been obtained by Rosvick et al. (1992), Mermilliod et al. (1997), and Queloz et al. (1998), and those authors identified a list of the candidate members that they considered confirmed by the high-resolution spectroscopy. For these outlying candidate members, to be included in Table 2 we require that the star be a radial velocity member from one of the above three surveys, or be indicated as having "no dip" in the Coravel cross-correlation (indicating rapid rotation, which at least for the later type stars is suggestive of membership). Geneva photometry of the Artyukhina stars considered as likely members was provided by Mermilliod et al. (1997). The magnitude limit of these surveys was not well-defined, but most of the Artyukhina and Pels stars are brighter than $V=13$.

Jones (1973) provided proper-motion membership probabilities for a large sample of proposed Pleiades members, and for a set of faint, red stars toward the Pleiades. A few star identification names from the sources considered by Jones appear in Table 2, including MT (McCarthy \& Treanor 1964), VM (van Maanen 1946), and ALR (Ahmed et al. 1965; Jones 1973).

The chronologically next significant source of new Pleiades candidate members was the flare star survey of the Pleiades conducted at several observatories in the 1960s, and summarized in Haro et al. (1982, hereafter HCG). The logic behind these surveys was that even at $100 \mathrm{Myr}$, late-type dwarfs have relatively frequent and relatively high-luminosity flares (as demonstrated by Johnson \& Mitchell 1958 having detected two flares during their photometric observations of the Pleiades), and therefore wide area, rapid cadence imaging of the Pleiades at blue wavelengths should be capable of identifying low-mass cluster members. However, such surveys also will detect relatively young field dwarfs, and therefore it is best to combine the flare star surveys with proper motions. 
Dedicated proper-motion surveys of the HCG flare stars were conducted by Jones (1981) and Stauffer et al. (1991), with the latter also providing photographic VI photometry (Kron system). Photoelectric photometry for some of the HCG stars have been reported in Stauffer (1982, 1984), Stauffer \& Hartmann (1987), and Prosser et al. (1991). High-resolution spectroscopy of many of the HCG stars is reported in Stauffer (1984), Stauffer \& Hartmann (1987), and Terndrup et al. (2000). Because a number of the papers providing additional observational data for the flare stars were obtained prior to 1982, we also include in Table 2 the original flare star names that were derived from the observatory where the initial flare was detected. Those names are of the form of an initial letter indicating the observatory - A (Asiago), B (Byurakan), K (Konkoly), T (Tonantzintla) — followed by an identification number.

Stauffer et al. (1991) conducted two proper-motion surveys of the Pleiades over an approximately $4^{\circ} \times 4^{\circ}$ region of the cluster based on plates obtained with the Lick $20^{\prime \prime}$ astrographic telescope. The first survey was essentially unbiased, except for the requirement that the stars fall approximately in the region of the $V$ versus $V-I$ color-magnitude diagram where Pleiades members should lie. Candidate members from this survey are designated by SK numbers. The second survey was a proper-motion survey of the HCG stars. Photographic VI photometry of all the stars was provided as well as proper-motion membership probabilities. Photoelectric photometry for some of the candidate members was obtained as detailed above in the section on the HCG catalog stars. The faint limit of these surveys is about $V=18$.

Hambly et al. (1991) provided a significantly deeper, somewhat wider area proper-motion survey, with the faintest members having $V \simeq 20$ and the total area covered being of order $25 \mathrm{deg}^{2}$. The survey utilized red sensitive plates from the Palomar and UK Schmidt telescopes. Due to incomplete coverage at one epoch, there is a vertical swath slightly east of the cluster center where no membership information is available. Stars from this survey are designated by their HHJ numbers. Hambly et al. (1993) provide RI photographic photometry on a natural system for all of their candidate members, plus photoelectric Cousins $R I$ photometry for a small number of stars and $J H K$ photometry for a larger sample. Some spectroscopy to confirm membership has been reported in Stauffer et al. (1994, 1995, 1999), Oppenheimer et al. (1997), and Steele et al. (1995), although for most of the HHJ stars there is no spectroscopic membership confirmation.

Pinfield et al. (2000) provide the deepest wide-field proper-motion survey of the Pleiades. That survey combines CCD imaging of $6 \mathrm{deg}^{2}$ of the Pleiades obtained with the Burrell Schmidt telescope (as five separate, nonoverlapping fields near but outside the cluster center) with deep photographic plates that provide the first epoch positions. Candidate members are designated by BPL numbers (for Burrell Pleiades), with the faintest stars having $I \simeq 19.5$, corresponding to $V>23$. Only the stars brighter than about $I=17$ have sufficiently accurate proper motions to use to identify Pleiades members. Fainter than $I=17$, the primary selection criteria are that the star fall in an appropriate place in both an $I$ versus $I-Z$ and an $I$ versus $I-K$ CMD.

Adams et al. (2001) combined the 2MASS and digitized POSS databases to produce a very wide area proper-motion survey of the Pleiades. By design, that survey was very inclusive - covering the entire physical area of the cluster and extending to the hydrogenburning mass limit. However, it was also very "contaminated," with many suspected nonmembers. The catalog of possible members was not published. We have therefore not included stars from this study in Table 2; we have used the proper-motion data from Adams et al. (2001) to help decide cases where a given star has ambiguous membership data from the other surveys.

Deacon \& Hambly (2004) provided another deep and very wide area proper-motion survey of the Pleiades. The survey covers a circular area of approximately $5^{\circ}$ radius to $R \sim 20$, or $V \sim 22$. Candidate members are designated by "DH." Deacon \& Hambly (2004) also provide membership probabilities based on proper motions for many candidate cluster members from previous surveys. For stars where Deacon \& Hambly (2004) derive $P<0.1$ and where we have no other proper-motion information or where another proper-motion survey also finds low membership probability, we exclude the star from our catalog. For cases where two of our propermotion catalogs differ significantly in their membership assessment, with one survey indicating the star is a probable member, we retain the star in the catalog as the conservative choice. Examples of the latter where Deacon \& Hambly (2004) derive $P<0.1$ include HII 1553, HII 2147, HII 2278, and HII 2665-all of which we retain in our catalog because other surveys indicate these are highprobability Pleiades members.

\section{A2. PHOTOMETRY}

Photometry for stars in open cluster catalogs can be used to help confirm cluster membership and to help constrain physical properties of those stars or of the cluster. For a variety of reasons, photometry of stars in the Pleiades has been obtained in a panoply of different photometric systems. For our own goals, which are to use the photometry to help verify membership and to define the Pleiades single-star locus in color-magnitude diagrams, we have attempted to convert photometry in several of these systems to a common system (Johnson $B V$ and Cousins $I$ ). We detail below the sources of the photometry and the conversions we have employed.

Photoelectric photometry of Pleiades members dates back to at least 1921 Cummings (1921). However, as far as we are aware the first "modern" photoelectric photometry for the Pleiades, using a potassium hydride photoelectric cell, is that of Calder \& Shapley (1937). Eggen (1950) provided photoelectric photometry using a 1P21 phototube (but calibrated to a no-longer-used photographic system) for most of the known Pleiades members within $1^{\circ}$ of the cluster center and with magnitudes $<11$. The first phototube photometry of Pleiades stars calibrated more-or-less to the modern $U B V$ system was provided by Johnson \& Morgan (1951). An update of that paper, and the oldest photometry included here was reported in Johnson \& Mitchell (1958), which provided UBV Johnson system photometry for a large sample of HII and Trumpler candidate Pleiades members. Iriarte (1967) later reported Johnson system $V-I$ colors for most of these stars. We have converted Iriarte's $V-I$ photometry to estimated Cousins $V-I$ colors using a formula from Bessell (1979):

$$
V-I(\text { Cousins })=0.778 \mathrm{~V}-I(\text { Johnson })
$$




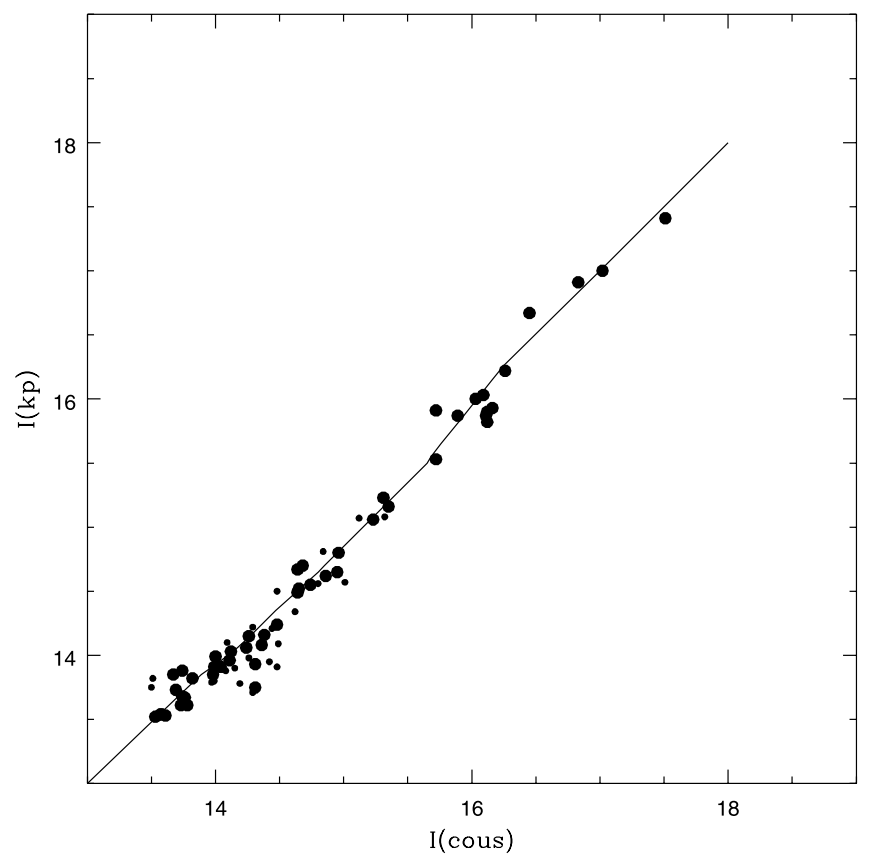

FIG. 21. - Calibration derived relating $I_{k p}$ from Pinfield et al. (2000) and $I_{\mathrm{C}}$. The dots represent stars for which we have both $I_{k p}$ and $I_{\mathrm{C}}$ measurements $(s m a l l$ dots: photographic $I_{\mathrm{C}}$; large dots: photoelectric $I_{\mathrm{C}}$ ), and the solid line indicates the piecewise linear fit we use to convert the $I_{k p}$ values to $I_{\mathrm{C}}$ for stars for which we only have $I_{k p}$.

BVRI photometry for most of the Hertzsprung members fainter than $V=10$ has been published by Stauffer $(1980,1982,1984)$ and Stauffer \& Hartmann (1987). The $B V$ photometry is Johnson system, whereas the RI photometry is on the Kron system. The Kron $V-I$ colors were converted to Cousins $V-I$ using a transformation provided by Bessell \& Weis (1987):

$$
V-I(\text { Cousins })=0.227+0.9567(V-I)_{k}+0.0128(V-I)_{k}^{2}-0.0053(V-I)_{k}^{3}
$$

Other Kron system $V-I$ colors have been published for Pleiades candidates in Stauffer et al. (1991, photographic photometry) and in Prosser et al. (1991). These Kron-system colors have also been converted to Cousins $V-I$ using the above formula.

Johnson/Cousins UBVR photometry for a set of low-mass Pleiades members was provided by Landolt (1979). We only use the $B V$ magnitudes from that study. Additional Johnson system $U B V$ photometry for small numbers of stars is provided in Robinson \& Kraft (1974), Messina (2001), and Jones (1973).

Van Leeuwen et al. (1987) provided Walraven VBLUW photometry for nearly all of the Hertzsprung members brighter than $V \sim 13.5$ and for the Pels candidate members. Van Leeuwen provided an estimated Johnson $V$ derived from the Walraven $V$ in his tables. We have transformed the Walraven $V-B$ color into an estimate of Johnson $B-V$ using a formula from Rosvick et al. (1992):

$$
B-V(\text { Johnson })=2.571(V-B)-1.02(V-B)^{2}+0.5(V-B)^{3}-0.01
$$

Hambly et al. (1993) provided photographic VRI photometry for all of the HHJ candidate members and VRI Cousins photoelectric photometry for a small fraction of those stars. We took all of the HHJ stars with photographic photometry for which we also have photoelectric $V I$ photometry on the Cousins system, and plotted $V($ Cousins) versus $V(\mathrm{HHJ})$ and $I($ Cousins) versus $I(\mathrm{HHJ})$. While there is some evidence for slight systematic departures of the HHJ photographic photometry from the Cousins system, those departures are relatively small and we have chosen simply to retain the HHJ values and treat them as Cousins system.

Pinfield et al. (2000) reported their $I$ magnitudes in an instrumental system that they designated as $I_{k p}$. We identified all BPL candidate members for which we had photoelectric Cousins $I$ estimates, and plotted $I_{k p}$ versus $I_{\mathrm{C}}$. Figure 21 shows this correlation, and the piecewise linear fit we have made to convert from $I_{k p}$ to $I_{\mathrm{C}}$. Our catalog lists these converted $I_{\mathrm{C}}$ measures for the BPL stars for which we have no other photoelectric $I$ estimates.

Deacon \& Hambly (2004) derived $R I$ photometry from the scans of their plates and calibrated that photometry by reference to published photometry from the literature. When we plotted their the difference between their $I$-band photometry and literature values (where available), we discovered a significant dependence on right ascension. Unfortunately, because the DH survey extended over larger spatial scales than the calibrating photometry, we could not derive a correction that we could apply to all the DH stars. We therefore developed the following indirect scheme. We used the stars for which we have estimated $I_{\mathrm{C}}$ magnitudes (from photoelectric photometry) to define the relation between $J$ and $\left(I_{\mathrm{C}}-J\right)$ for Pleiades members. For each DH star, we combined that relation and the 2MASS $J$ magnitude to yield a predicted $I_{\mathrm{C}}$. Figure 22 shows a plot of the difference of this predicted $I_{\mathrm{C}}$ and $I(\mathrm{DH})$ with right ascension. The solid line shows the relation we adopt. Figure 23 shows the relation between the corrected $I(\mathrm{DH})$ values and Table $2 I_{\mathrm{C}}$ measures from photoelectric sources. There is still a significant amount of scatter, but the corrected $I(\mathrm{DH})$ photometry appears to be accurately calibrated to the Cousins system. 


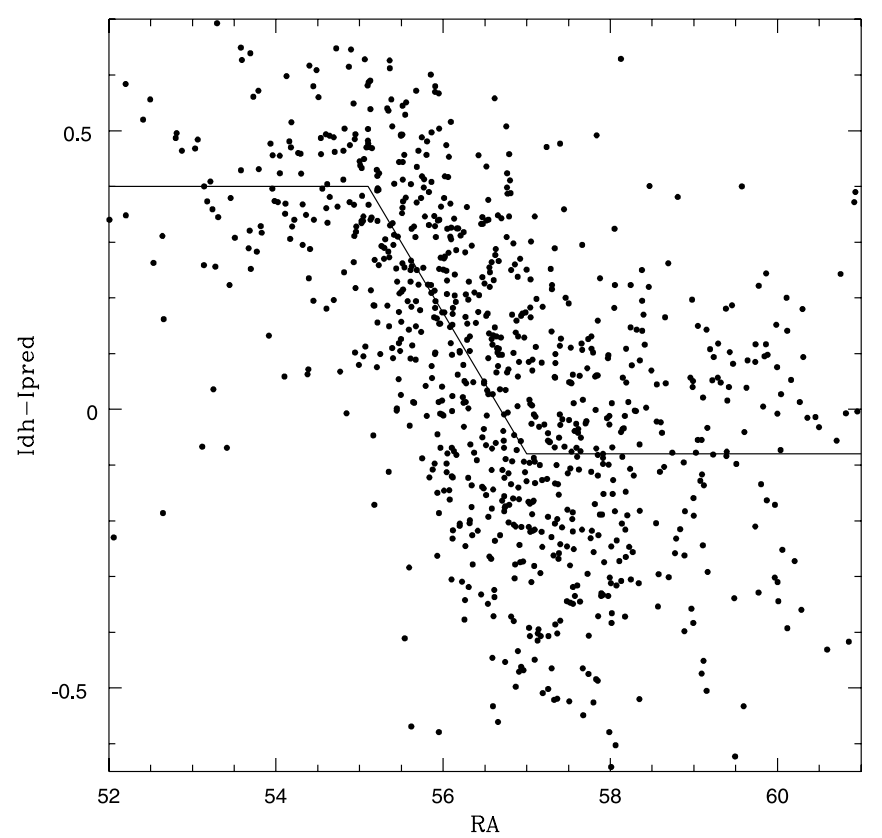

Fig. 22. - Difference between the predicted $I_{\mathrm{C}}$ and Deacon \& Hambly (2004) $I$ magnitude as a function of right ascension for the DH stars. No obvious dependence is present vs. declination.

In a very few cases (specifically, just five stars), we provide an estimate of $I_{\mathrm{C}}$ based on data from a wide-area CCD survey of Taurus obtained with the Quest-2 camera on the Palomar 48 inch Samuel Oschin telescope (Slesnick et al. 2006). That survey calibrated their photometry to the Sloan $i$ system, and we have converted the Sloan $i$ magnitudes to $I_{\mathrm{C}}$. We intend to make more complete use of the Quest-2 data in a subsequent paper.

When we have multiple sources of photometry for a given star, we consider how to combine them. In most cases, if we have photoelectric data, that is given preference. However, if we have photographic $V$ and $I$, and only a photoelectric measurement for $I$, we do not replace the photographic $I$ with the photoelectric value because these stars are variable and the photographic measurements are at least in some cases from nearly simultaneous exposures. Where we have multiple sources for photoelectric photometry, and no strong reason to favor one measurement or set of measurements over another, we have averaged the photometry for a given star. In most cases where we have multiple photometry the individual measurements agree reasonably well but with the caveat that the Pleiades low-mass stars are in many cases heavily spotted and "active" chromospherically and hence are photometrically variable. In a few cases, even given the expectation that spots and other phenomena may affect the photometry, there seems to be more discrepancy between reported $V$ magnitudes than we expect. We note two such cases here. We suspect these results indicate that at least some of the Pleiades low-mass stars have long-term photometric variability larger than their short period (rotational) modulation.

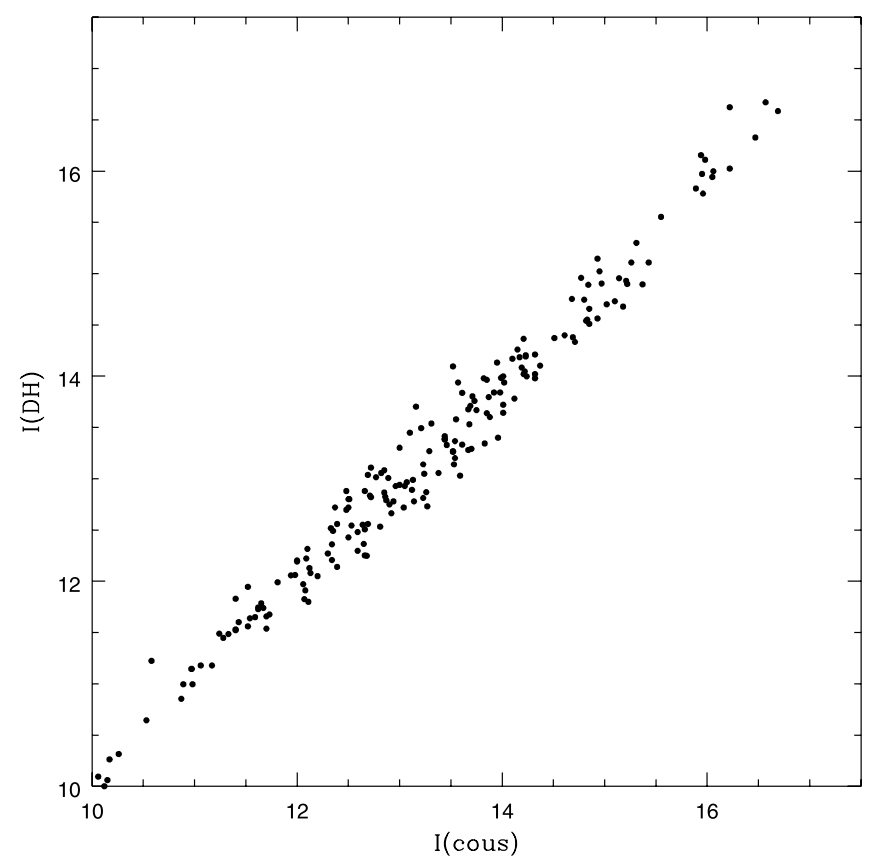

FIG. 23. - Comparison of the recalibrated DH $I$ photometry with estimates of $I_{\mathrm{C}}$ for stars in Table 2 with photoelectric data. 
HII 882 has at least four presumably accurate $V$ magnitude measurements reported in the literature. Those measures are $V=12.66$ Johnson \& Mitchell (1958); $V=12.95$ Stauffer (1982); $V=12.898$ van Leeuwen et al. (1986); and $V=12.62$ Messina (2001).

HII 345 has at least three presumably accurate $V$ magnitude measurements. Those measurements are $V=11.65$ Landolt (1979); $V=11.73$ van Leeuwen et al. (1986); $V=11.43$ Messina (2001).

At the bottom of Table 2, we provide a key to the source(s) of the optical photometry provided in the table.

\section{REFERENCES}

Adams, J., Stauffer, J., Monet, D., Skrutskie, M., \& Beichman, C. 2001, AJ, 121,2053

Ahmed, F., Lawrence, L., \& Reddish, V. 1965, Publ. R. Obs. Edinburgh, 3, 187

Allen, L., et al. 2004, ApJS, 154, 363

Artymowicz, P., \& Clampin, M. 1997, ApJ, 490, 863

Artyukhina, N. 1969, Soviet Astron., 12, 987

Artyukhina, N., \& Kalinina, E. 1970, Trudy Sternberg Astron. Inst., 40, 3

Baraffe, I., Chabrier, G., Allard, F., \& Hauschildt, P. 1998, A\&A, 337, 403

Bessell, M. 1979, PASP, 91, 589

Bessell, M., \& Weis, E. 1987, PASP, 99, 642

Bihain, G., et al. 2006, A\&A, 458, 805

Breger, M. 1986, ApJ, 309, 311

Calder, W., \& Shapley, H. 1937, Ann. Ast. Obs. Harvard College, 105, 453

Chabrier, G., Baraffe, I., Allard, F., \& Hauschildt, P. 2000, ApJ, 542, 464

Cruz, K., et al. 2007, AJ, 133, 439

Cummings, E. 1921, PASP, 33, 214

Deacon, N., \& Hambly, N. 2004, A\&A, 416, 125

Eggen, O. 1950, ApJ, 111, 81

Federman, S., \& Willson, R. 1984, ApJ, 283, 626

Festin, L. 1998, A\&A, 333, 497

Gorlova, N., et al. 2006, ApJ, 649, 1028

Hambly, N., Hawkins, M. R. S., \& Jameson, R. 1991, MNRAS, 253, 1 1993, A\&AS, 100, 607

Haro, G., Chavira, E., \& Gonzalez, G. 1982, Bol. Inst. Tonantzintla, 3, 1

Herbig, G., \& Simon, T. 2001, AJ, 121, 3138

Hertzsprung, E. 1923, Mem. Danish Acad. 4, 4 . 1947, Ann. Leiden Obs., 191, 1

Iriarte, B. 1967, Bol. Obs. Tonantzintla Tacubaya, 4, 79

Jameson, R., \& Skillen, I. 1989, MNRAS, 239, 247

Jarrett, T., Chester, T., Cutri, R., Schneider, S., Skrutskie, M., \& Huchra, J. 2000, AJ, 119, 2498

Jeffries, R. D., \& Oliveira, J. 2005, MNRAS, 358, 13

Johnson, H. L., \& Mitchell, R. I. 1958, ApJ, 128, 31 (JM)

Johnson, H. L., \& Morgan, W. W. 1951, ApJ, 114, 522

Jones, B. F. 1973, A\&AS, 9, 313 1981, AJ, 86, 290

Landolt, A. 1979, ApJ, 231, 468

Makovoz, D., \& Marleau, F. 2005, PASP, 117, 1113

McCarthy, M., \& Treanor, P. 1964, Ric. Astron., 6, 535

Mermilliod, J.-C., Bratschi, P., \& Mayor, M. 1997, A\&A, 320, 74

Messina, S. 2001, A\&A, 371, 1024

Meynet, G., Mermilliod, J.-C., \& Maeder, A. 1993, A\&AS, 98, 477

Oppenheimer, B., Basri, G., Nakajima, T., \& Kulkarni, S. 1997, AJ, 113, 296

Patten, B., et al. 2006, ApJ, 651, 502
Pinfield, D., Hodgkin, S., Jameson, R., Cossburn, M., Hambly, N., \& Devereux, N. 2000, MNRAS, 313, 347

Pritchard, R. 1884, MNRAS, 44, 355

Prosser, C., Stauffer, J., \& Kraft, R. 1991, AJ, 101, 1361

Queloz, D., Allain, S., Mermilliod, J.-C., Bouvier, J., \& Mayor, M. 1998, A\&A, 335,183

Raboud, D., \& Mermilliod, J.-C. 1998, A\&A, 329, 101

Rieke, G., \& Lebofsky, M. 1985, ApJ, 288, 618

Robinson, E. L., \& Kraft, R. P. 1974, AJ, 79, 698

Rosvick, J., Mermilliod, J., \& Mayor, M. 1992, A\&A, 255, 130

Siess, L., Dufour, E., \& Forestini, M. 2000, A\&A, 358, 593

Skrutskie, M., et al. 2006, AJ, 131, 1163

Slesnick, C., Carpenter, J., Hillenbrand, L., \& Mamajek, E. 2006, AJ, 132, 2665

Soderblom, D. R., Jones, B. R., Balachandran, S., Stauffer, J. R., Duncan, D. K., Fedele, S. B., \& Hudon, J. 1993, AJ, 106, 1059

Soderblom, D., Nelan, E., Benedict, G., McArthur, B., Ramirez, I., Spiesman, W., \& Jones, B. 2005, AJ, 129, 1616

Stauffer, J. 1980, AJ, 85, 1341 1982, AJ, 87, 1507

1984, ApJ, 280, 189

Stauffer, J. R., Caillault, J.-P., Gagne, M., Prosser, C. F., \& Hartmann, L. W. 1994, ApJS, 91, 625

Stauffer, J., Hamilton, D., Probst, R., Rieke, G., \& Mateo, M. 1989, ApJ, 344, L21

Stauffer, J. R., \& Hartmann, L. W. 1987, ApJ, 318, 337

Stauffer, J. R., Hartmann, L. W., Soderblom, D. R., \& Burnham, N. 1984, ApJ, 280, 202

Stauffer, J., Klemola, A., Prosser, C., \& Probst, R. 1991, AJ, 101, 980

Stauffer, J. R., Liebert, J., \& Giampapa, M. 1995, AJ, 109, 298

Stauffer, J. R., et al. 1999, ApJ, 527, 219 . 2005, AJ, 130, 1834

Steele, I., et al. 1995, MNRAS, 272, 630

Terlevich, E. 1987, MNRAS, 224, 193

Terndrup, D. M., Stauffer, J. R., Pinsonneault, M. H., Sills, A., Yuan, Y., Jones, B. F., Fischer, D., \& Krishnamurthi, A. 2000, AJ, 119, 1303

Trumpler, R. J. 1921, Lick Obs. Bull., 10, 110

van Leeuwen, F., Alphenaar, P., \& Brand, J. 1986, A\&AS, 65, 309

van Leeuwen, F., Alphenaar, P., \& Meys, J. J. M. 1987, A\&AS, 67, 483

van Maanen, A. 1946, ApJ, 103, 289

Werner, M., et al. 2004, ApJS, 154, 309

White, R. E. 2003, ApJS, 148, 487

White, R. E., \& Bally, J. 1993, ApJ, 409, 234 